\title{
7 Medienprävention- und intervention
}

Wurden in den vorherigen Kapiteln die Mediennutzung selbst (Kapitel 2 und Kapitel 5) sowie die Folgen einer problematischen Mediennutzung im Sinne einer zeitlich exzessiven Mediennutzung oder einer inhaltlich problematischen Mediennutzung (Kapitel 3, Kapitel 4 und Kapitel 6) thematisiert, soll in vorliegendem Kapitel das durchgeführte Medienunterrichtsprogramm des Berliner Längsschnitts Medien in den Mittelpunkt gerückt werden. Wie war die Akzeptanz des Programmes bei den Berliner Schülerinnen und Schülern, wie die Wirkungen auf die kindliche Mediennutzung? Ferner ist von Interesse, ob auch bezüglich der oben betrachteten Variablen Schulleistung, Gewaltverhalten, Computerspielabhängigkeit sowie Fettleibigkeit Effekte des durchgeführten Programmes beobachtet werden konnten. Eine kurze Beschreibung des Unterrichtsprogrammes des Berliner Längsschnitts Medien findet sich in Kapitel 1.3. Zur genauen Konzeption des Medienunterrichtsprogrammes siehe Kleimann (2011).

Im Folgenden sollen die theoretischen Grundlagen und empirische Forschungsergebnisse zu Medieninterventions- und Medienpräventionsprogrammen nur überblicksartig dargestellt werden. Eine ausführliche Beschreibung dieser sowie eine theoretische Einordnung des durchgeführten Medienunterrichtsprogramms des Berliner Längsschnitts Medien findet sich bei Kleimann (2011), der sich ausschließlich und umfassend mit dem durchgeführten Medienunterrichtskonzept des Berliner Längsschnitts Medien befasst; die Effekte des medienpädagogischen Unterrichts auf Gewaltverhalten und Fettleibigkeit werden dort jedoch nicht abgehandelt. Nach diesem kurzen theoretischen Überblick (Kapitel 7.1) werden die Ergebnisse des Berliner Längsschnitts Medien präsentiert (Kapitel 7.2) und mit Ersterem in Beziehung gesetzt (Kapitel 7.3).

\subsection{Theoretische Grundlagen und Literaturüberblick - Medienprävention- und intervention}

Trotz der belegten Zusammenhänge zwischen einer inhaltlich problematischen wie zeitlich exzessiven Mediennutzung und schlechteren Schulleistungen (vgl. Kapitel 3), erhöhtem Gewaltverhalten (vgl. Kapitel 4) sowie Fettleibigkeit (vgl. Kapitel 6) wurden die Auswirkungen auf Schulleistungen nie zur Begründung und Etablierung unterschiedlicher Präventionsprogramme herangezogen. Der Begründungszusammenhang bestehender Medienpräventions- und Interventionsmaßnahmen beschränkt sich bisher fast ausschließlich auf Gewaltprävention und 
allenfalls noch Gesundheitsförderung; das Ziel der Verbesserung schulischer Leistungen durch die Reduktion von Dauer und Anteil problematischer Inhalte dagegen ist Neuland.

Grundsätzlich können somit Medieninterventions- und Medienpräventionsprogramme, für die positive Interventionseffekte berichtet wurden, in zwei Gruppen unterteilt werden: Programme mit dem Ziel Gewaltverhalten zu verhindern oder zu reduzieren sowie Programme zur Förderung des Gesundheitsverhaltens (diese beinhalten das Essverhalten, Fitnesstraining oder auch Drogenaufklärung). Die Thematisierung problematischer Mediennutzung (vor allem von Fernsehinhalten und Fernsehnutzungszeiten) dient hierbei jeweils nur als ein Weg, der aufgrund der Zusammenhänge von Mediennutzung mit dem jeweils zu beeinflussenden Konstrukt (Fettleibigkeit, Gewaltverhalten) eingeschlagen wird. So sollen Kinder und Jugendliche durch eine bewusste Mediennutzung, welche zum Beispiel lange Nutzungszeiten vermeidet, in ihrem Sozial- oder Gesundheitsverhalten gestärkt werden, oder es wird versucht, zur Vermeidung etwa von Fettleibigkeit, Kinder und Jugendliche für bestimmte Überzeugungsstrategien, hier zum Beispiel von Werbebotschaften, zu sensibilisieren. ${ }^{215}$

In den unterschiedlichen Interventionsstrategien sollen mittels Verhaltenstrainings oder mittels verschiedener Verhaltensrichtlinien (Fernsehzeitlimits oder Reduktion von Mediengewaltinhalten) Medienkompetenz gefördert oder problematisches Verhalten verhindert werden (vgl. Kleimann, 2011; Kunczik \& Zipfel, 2004). Sie lassen sich nach den Hauptbeteiligten, über deren Vermittlung diese Ziele bei den Kindern und Jugendlichen erreicht werden sollen, nach Cantor und Wilson (2003; vgl. Kunczik \& Zipfel, 2004; Mößle et al., 2010) in drei Bereiche unterteilen: Eltern oder andere Erziehende (im Kontext von Maßnahmen der häuslichen Mediennutzung), Lehrkräfte (im Rahmen von schulbasierten Maßnahmen) sowie die Medien selbst (Maßnahmen die Interventionsziele über die Massenmedien verbreiten).

Für alle drei Strategien liegen Forschungsergebnisse vor, die einen bedeutsamen Interventionseffekt berichten können; Maßnahmen aus dem Bereich der Medien selbst sowie elterliche Medienerziehungsprogramme kämpfen aber oft

215 Mit einer derartigen inhaltlichen Fokussierung lassen sich die durchgeführten Präventionsmaßnahmen somit in der Definition von Caplan (1964) den primären und sekundären Präventionsmaßnahmen zuordnen. Während primäre Präventionsmaßnahmen eine Inzidenz einer bestimmten Erkrankung oder allgemeiner einer negativen oder als negativ wahrgenommenen Handlung oder eines derartigen Zustandes verhindern sollen, sollen sekundäre Präventionsmaßnahmen die Prävalenzraten oder die Dauer verringern (vgl. Caplan, 1964; Kleimann, 2011). Tertiäre Präventionsmaßnahmen dienen schließlich dazu die Auswirkungen zu verringern (vgl. Caplan, 1964). Auf den Bereich der Mediennutzung übertragen wären beispielsweise Maßnahmen zur Reduktion medienbezogenen Risikoverhaltens primäre, Maßnahmen zur Korrektur bereits bestehender problematischer Mediennutzungsmuster sekundäre sowie Einrichtungen zur ambulanten Nachsorge der Computerspielabhängigkeit tertiäre Präventionsmaßnahmen (vgl. Kleimann, 2011). 
mit dem Problem der externen Validität. So fassen Kunczik und Zipfel (2004) in ihrem allgemeinen Überblick „Gewalt und Medien“ die Forschungsergebnisse zu Interventionseffekten von Maßnahmen zu Mediengewalt und Gewaltverhalten wie folgt zusammen: Für medial vermittelte Anti-Gewalt-Botschaften, vor allem für Programme welche eindeutige Botschaften enthalten und das Leiden der Opfer thematisieren, können nur kurzzeitige Veränderungen von gewaltbezogenen Einstellungen bzw. eine größere Empathie bezüglich des Leidens der Opfer beobachtet werden, nicht jedoch eine tatsächliche Verminderung des Gewaltverhaltens (vgl. auch Cantor \& Wilson, 2003).

Darüber hinaus könnten elterliche Strategien und Erziehungsbemühungen zwar in einigen experimentellen Studien gewaltbezogene Einstellungen und gewaltbezogenes Verhalten von Kindern beeinflussen (vgl. Nathanson, 2004; Nathanson \& Yang, 2003), im Setting des wirklichen Lebens sei eine erfolgreiche elterliche Einflussnahme jedoch an bestimmte Voraussetzungen gebunden: eine aktive elterliche Medienerziehung mit Reflektion der konsumierten Medieninhalte, einen hohen Bildungsstand der Eltern, eine dauerhafte Anwesenheit zu Hause, ein gutes elterliches Erziehungs- und Unterstützungsverhalten (insbesondere im schulischen Bereich), sowie ein hohes Bewusstsein für mögliche negative Medienwirkungen (vgl. Kunczik \& Zipfel, 2004; Valkenburg et al., 1999; Warren, 2001; Warren et al., 2002; Mößle et al., 2007; Mößle et al., 2010). Darüber hinaus seien Präventionsmaßnahmen in diesem Bereich umso effektiver, je jünger die Kinder sind (vgl. Kunczik \& Zipfel, 2004).

Vor dem Hintergrund dieser Ergebnisse kommt insbesondere schulbasierten Präventionsprogrammen eine besondere Bedeutung zu, da diese zum einen die Möglichkeit bieten auch Gruppen zu erreichen, die sonst nicht von Präventionsprogrammen profitieren können, so zum Beispiel Kinder aus Familien mit niedrigem Bildungsniveau und geringer elterlicher Zuwendung. Zum anderen kann man sich in diesem Bereich auch mögliche Langzeiteffekte erhoffen. Insgesamt betrachtet konnten von den wenigen veröffentlichten schulischen Medieninterventions- und Medienpräventionsprogrammen, die gewaltbezogene Einstellungen sowie das Gewaltverhalten von Kindern und Jugendlichen beeinflussen wollten, vor allem jene Erfolge verzeichnen, welche mehr als nur eine thematische Einheit beinhalteten und ebenso wiederholt über einen längeren Zeitraum durchgeführt wurden (vgl. Cantor \& Wilson, 2003; Kunczik \& Zipfel, 2004; Rosenkoetter, Rosenkoetter, Ozretich \& Acock, 2004; siehe auch Mößle et al., 2010; Kleimann, 2011). Schulische Programme, welche die Reflexion möglicher negativer Wirkungen einer Gewaltmediennutzung fokussierten, hatten zwar einen bedeutsamen Effekt auf das Gewaltverhalten der Schülerinnen und Schüler, konnten jedoch nicht deren Faszination für Gewaltmedien sowie die Nutzungshäufigkeit von Gewaltmedien bedeutsam beeinflussen (Kunczik \& Zipfel, 2004; 
Rosenkoetter et al., 2004; siehe auch Mößle et al., 2010; Kleimann, 2011). Ferner gibt es Belege dafür, dass schulbasierte Maßnahmen, die allein eine Reduktion des täglichen Medienkonsums zum Ziel hatten, ohne Gewalt explizit zu thematisieren, ebenso das Gewaltverhalten der Schülerinnen und Schüler reduzieren konnten (vgl. Robinson et al., 2001; siehe Mößle et al., 2010). Langfristige Effekte schulbasierter Medieninterventions- und Medienpräventionsprogramme sind somit immer auch abhängig davon, inwiefern das Mediennutzungsverhalten der Kinder und Jugendlichen langfristig verändert werden kann (vgl. Kleimann, 2011).

Zahlreiche schulbasierte Medieninterventions- und Medienpräventionsmaßnahmen des letzten Jahrzehnts versuchten im Rahmen von Programmen zum Gesundheitsverhalten und zur Reduktion von Fettleibigkeit von Kindern und Jugendlichen deren Mediennutzung positiv zu beeinflussen: Kinder und Jugendliche, so der Ansatz, sollten zu einer moderaten Nutzung elektronischer Bildschirmmedien angeleitet werden sowie die Folgen der Werbung sowie medial vermittelter Stereotypen auf ihr Gesundheitsverhalten erkennen lernen (vgl. Kleimann, 2011; Mößle et al., 2010). So konnte Robinson (1999; vgl. Robinson et al., 2001) in einem randomisierten Kontrollgruppendesign nachweisen, dass insbesondere die Kombination aus schulbasierten Medienunterrichtseinheiten in Verbindung mit elektronischen Nutzungszeitreglern heimischer Mediennutzung (vgl. Epstein et al., 2008) geeignet ist, sowohl Bildschirmmediennutzungszeiten von Grundschulkindern zu verringern, als auch deren Gesundheitsverhalten zu verbessern sowie ihr Körpergewicht zu reduzieren - und dies ohne eine explizite Thematisierung des Gesundheitsverhaltens (vgl. Kleimann, 2011; Mößle et al., 2010). Kline (2005) konnte vergleichbare Effekte in einem ähnlichen Design auch ohne die Verwendung elektronischer Nutzungszeitregler heimischer Mediennutzung in den elterlichen Haushalten berichten; Sie fanden kurzzeitige Effekte auf das Mediennutzungs- und Freizeitverhalten kanadischer Grundschülerinnen und Grundschüler (in der Veröffentlichung dieser Studie werden die berichteten Unterschiede zwischen Kontroll- und Interventionsgruppe allerdings lediglich deskriptiv dargestellt).

Zusammengefasst können schulbasierte Medieninterventions- und Medienpräventionsmaßnahmen - unabhängig von ihrer ursprünglichen Intention - als vielversprechende Ansätze für ein bewusstes und kontrolliertes kindliches und jugendliches Mediennutzungsverhalten erachtet werden.

Neben diesen Programmen, die allesamt der Health-Promotion-Perspektive zugeordnet werden können, welche Forschungsansätze aus der Public-HealthForschung bzw. der Gesundheitswissenschaft, der psychologischen Gesundheitsförderung, der Medizin und der Medienwissenschaft vereint (vgl. Kleimann, 2011), gibt es zudem medienpädagogische Ansätze, welche die Förderung der 
Medienkompetenz von Kindern und Jugendlichen in den Fokus der präventiven Bemühungen stellen. Medienkompetenz kann dabei beispielsweise nach Baacke (1997) als Ziel medienpädagogischen Handelns formuliert und in vier verschiedene Dimensionen eingeteilt werden: Mediengestaltung, Medienkritik, Medienkunde und Mediennutzung. In einer elaborierteren Definition nach Groeben (2004) würde Medienkompetenz beispielsweise das Medienwissen/Medialitätsbewusstsein, medienspezifische Rezeptionsmuster, medienbezogene Genussfähigkeit, medienbezogene Kritikfähigkeit, Selektion/Kombination von Mediennutzung, Partizipationsmuster und Anschlusskommunikation ausmachen.

„Ein Hauptproblem aller pädagogischen Konzepte, die sich auf Medienkompetenz, ihre Vermittlung und Förderung berufen, ist die große Unschärfe des Begriffs. Durch die Breite seines Geltungsanspruches, durch die Vielzahl und ständige Ausdifferenzierung als wichtig erachteter Teildimensionen verliert das Konzept der Medienkompetenz zunehmend an Kontur, empirischer Messbarkeit und letztlich auch - von seiner rein formelhaften Verwendung einmal abgesehen - an Bedeutung für die konkret-medienpädagogische Praxis“ (Kleimann, 2011, S. 163).

Neben dieser grundsätzlichen theoretischen Kritik ist anzumerken, dass Forschungsergebnisse vor allem zur Verbreitung von Programmen zur Förderung von Medienkompetenz (vgl. Gysbers, 2008; Schneider, B., Scherer, Gonser \& Tiele, 2010; Six \& Gimmler, 2007; Tulodziecki \& Six, 2000) vorliegen und sich nur wenige evaluierte Programme zur expliziten Förderung der Medienkompetenz finden. Aus diesen Gründen und vor allem, da diese Ansätze für eine Einordnung eines medienpädagogischen Unterrichtsprogrammes, welches die Verhinderung problematischer Mediennutzungsmuster thematisiert, wenig gewinnbringend sind, soll auf eine separate Darstellung dieser Ergebnisse an dieser Stelle verzichtet werden (für eine ausführliche Darstellung und kritische Würdigung dieser Ansätze siehe Kleimann, 2011).

\subsection{Ergebnisse des Berliner Längsschnitt Medien - Evaluation des Medienunterrichts}

Zwischen den ersten vier Messzeitpunkten fanden innerhalb des Projektes Berliner Längsschnitt Medien jeweils Blöcke mit medienpädagogischem Unterricht statt. So wurde bereits parallel zur ersten Datenerhebung unter Berücksichtigung des Berliner Rahmenlehrplans sowie unter Mitwirkung der Lehrkräfte ein Unterrichtsprogramm für Medienerziehung in der Grundschule erarbeitet, welches besonders die Rolle von Medien im Alltag der Kinder beleuchtet und in den darauf folgenden Jahren jeweils für die neue Jahrgangsstufe erweitert wurde. Da dieses 
Unterrichtsprogramm nicht nur im Elternhaus, sondern auch im schulischen Kontext auf eine bestehende mediale Ausstattung und Nutzung trifft, wurden die teilnehmenden Lehrkräfte zu allen vier Messzeitpunkten zu Medienausstattung in Schule und Klasse sowie zur Nutzungshäufigkeit und didaktischen Eignung dieser Medien befragt. ${ }^{216}$ In Tabelle 81 sind die Ergebnisse der ersten Befragungswelle in der dritten Klasse sowie der fünften Befragungswelle in der sechsten Klasse dargestellt.

Tabelle 81. Medienausstattung in Schule und Klasse: Nutzung und didaktische Eignung.

\begin{tabular}{|c|c|c|c|c|c|c|c|}
\hline & PC & Laptop & Internet & Beamer & Video & DVD & TV \\
\hline \multicolumn{8}{|l|}{ 3. Klasse } \\
\hline An Schule vorhanden & $100 \%$ & $26.7 \%$ & $100 \%$ & $73.9 \%$ & $100 \%$ & $58.7 \%$ & $82.6 \%$ \\
\hline In Klasse vorhanden & $47.8 \%$ & - & $10.9 \%$ & - & $6.5 \%$ & $2.2 \%$ & $4.3 \%$ \\
\hline \multicolumn{8}{|l|}{ Nutzung } \\
\hline nie & $6.7 \%$ & $75.0 \% *$ & $39.5 \%$ & $70.4 \%$ & - & $25.0 \% *$ & $71.9 \%$ \\
\hline selten & $44.4 \%$ & $25.0 \% *$ & $57.9 \%$ & $22.2 \%$ & $92.3 \%$ & $70.0 \% *$ & $28.1 \%$ \\
\hline häufig & $48.9 \%$ & $-*$ & $2.6 \%$ & $7.4 \%$ & $7.7 \%$ & $5.0 \% *$ & - \\
\hline sehr häufig & - & $-*$ & - & - & - & $-*$ & - \\
\hline \multicolumn{8}{|l|}{ Didaktische Eignung } \\
\hline sehr schlecht & - & $4.5 \% *$ & - & - & - & - & $9.7 \%$ \\
\hline eher schlecht & $11.1 \%$ & $13.6 \% *$ & $13.2 \%$ & $6.7 \%$ & $2.5 \%$ & $9.7 \%$ & $58.1 \%$ \\
\hline eher gut & $64.4 \%$ & $68.2 \% *$ & $52.6 \%$ & $70.0 \%$ & $80.0 \%$ & $61.3 \%$ & $32.3 \%$ \\
\hline sehr gut & $24.4 \%$ & $13.6 \% *$ & $34.2 \%$ & $23.3 \%$ & $17.5 \%$ & $29.0 \%$ & - \\
\hline \multicolumn{8}{|l|}{ 6. Klasse } \\
\hline An Schule vorhanden & $100 \%$ & $47.5 \%$ & $97.5 \%$ & $87.5 \%$ & $95.0 \%$ & $97.5 \%$ & $82.5 \%$ \\
\hline In Klasse vorhanden & $57.5 \%$ & $10.0 \%$ & $55.0 \%$ & $10 \%$ & $15.0 \%$ & $15.0 \%$ & $10.0 \%$ \\
\hline \multicolumn{8}{|l|}{ Nutzung } \\
\hline nie & $2.6 \%$ & $25.0 \% *$ & $-\%$ & $19.4 \%$ & - & $5.7 \%$ & $39.3 \%$ \\
\hline selten & $33.3 \%$ & $37.5 \% *$ & $32.4 \%$ & $54.8 \%$ & $70.6 \%$ & $54.3 \%$ & $46.4 \%$ \\
\hline häufig & $56.4 \%$ & $25.0 \% *$ & $62.2 \%$ & $22.6 \%$ & $29.4 \%$ & $40.0 \%$ & $14.3 \%$ \\
\hline sehr häufig & $7.7 \%$ & $12.5 \% *$ & $5.4 \%$ & $3.2 \%$ & - & - & - \\
\hline \multicolumn{8}{|l|}{ Didaktische Eignung } \\
\hline sehr schlecht & - & - & - & - & - & - & $6.2 \%$ \\
\hline eher schlecht & $2.6 \%$ & $4.5 \%$ & $2.7 \%$ & - & $8.6 \%$ & $2.8 \%$ & $31.2 \%$ \\
\hline eher gut & $65.8 \%$ & $59.1 \%$ & $56.8 \%$ & $56.7 \%$ & $71.4 \%$ & $75.0 \%$ & $53.1 \%$ \\
\hline sehr gut & $31.6 \%$ & $36.4 \%$ & $40.5 \%$ & $43.3 \%$ & $20.0 \%$ & $22.2 \%$ & $9.4 \%$ \\
\hline
\end{tabular}

Anmerkung. * Angaben von weniger als 50\% der Lehrkräfte.

216 Welche Medien gibt es an der Schule bzw. im Klassenzimmer der an der Befragung teilnehmenden Klasse? Wie häufig werden diese genutzt und wie bewerten Sie im Allgemeinen den didaktischen Nutzen dieser Medien? 
In der dritten Klasse gaben die Lehrkräfte aller teilnehmenden Klassen an, dass an der Schule ein PC, ein Internetanschluss sowie ein Videorekorder vorhanden seien. An rund 83 Prozent der Schulen war zusätzlich ein TV-Gerät, an rund 74 Prozent der Schulen ein Beamer und an rund 59 Prozent der Schulen ein DVDPlayer vorhanden. Ein Laptop stand nach Auskunft der Lehrkräfte hingegen im November 2005 lediglich einem Viertel der Schulen zur Verfügung. Die Ausstattungsquote in den Klassenzimmern war erwartungsgemäß auf einem deutlich geringeren Niveau. Am häufigsten gab es mit 48 Prozent noch einen eigenen PC im Klassenzimmer sowie in rund einem Zehntel der Klassenzimmer zusätzlich einen Internetanschluss. $\mathrm{Zu}$ einem noch deutlich geringeren Prozentsatz waren die Klassenzimmer mit einem Video- oder TV-Gerät oder einem DVD-Player ausgestattet. In keiner der teilnehmenden Klassen gab es hingegen einen eigenen Laptop oder Beamer. Bis zur sechsten Klasse ist auch an den Schulen eine Ausstattungszunahme mit Mediengeräten, insbesondere mit Laptops (47.5\%), Beamern (87.5\%) und DVD-Playern (97.5\%), festzustellen, sodass annähernd allen Schulen eine mediale Vollausstattung zur Verfügung stand. Auch die Ausstattungsquote in den Klassenzimmern änderte sich innerhalb der drei Jahre in derselben Richtung. So gaben in der sechsten Klasse 58 Prozent der Lehrkräfte der teilnehmenden Klassen an, dass im Klassenzimmer ein PC vorhanden sei. In rund 55 Prozent der Klassenzimmer gab es zusätzlich einen Internetanschluss und auch bei der Ausstattung mit einem Laptop, einem Beamer, einem Videooder TV-Gerät sowie einem DVD-Player sind leichte Zunahmen zu verzeichnen. Am häufigsten genutzt wurde von den Lehrkräften in der dritten Klasse der PC, während die anderen Mediengeräte äußerst selten oder nie zum Einsatz kamen. Allen Mediengeräten, mit Ausnahme des TV-Geräts (68\% schlecht oder eher schlecht), wurde von den Lehrkräften zudem eine eher gute oder sehr gute didaktische Eignung attestiert. In der sechsten Klasse ist ein deutlich breiterer Einsatz der unterschiedlichen Medien festzustellen. So gaben die Lehrkräfte an neben dem PC auch das Internet, einen Beamer, einen DVD-Player oder ein Videogerät in der Klasse einzusetzen. Allen Mediengeräten, auch dem TV-Gerät (62 Prozent) wurde von den Lehrkräften zudem eine eher gute oder sehr gute didaktische Eignung bescheinigt.

In den Lehrerfragebögen der sechsten Klasse wurde über Ausstattung mit Mediengeräten in Schule und Klasse hinaus noch zusätzlich die schulische Reglementierung der Nutzung privater Mediengeräte abgefragt. ${ }^{217}$ An den meisten Schulen dürfen Handys zwar mitgebracht (94.9\%), aber nicht (87.2\%), nur in

217 Wie ist der Umgang mit privaten Mediengeräten in ihrer Schule geregelt? Handys, tragbare Spielkonsolen, MP3-Player: ,dürfen nicht in die Schule mitgebracht werden“, „dürfen zwar in die Schule mitgebracht, aber nicht benutzt werden“, „dürfen zwar in die Schule mitgebracht, aber nur in den Pausen genutzt werden“, „, bei uns ist das anders geregelt, und zwar: 
der Pause $(5.1 \%)$ oder nur in Notfällen (2.6\%) benutzt werden. Spielkonsolen dürfen hingegen an den meisten Schulen (70.3\%) nicht mitgebracht werden. Dürfen sie mitgebracht werden, können sie entweder gar nicht (10.8\%), nur in der Pause $(16.2 \%)$ oder nur im Freizeitbereich $(2.7 \%)$ zum Spielen verwendet werden. MP3-Player dürfen an einem Drittel (67.6\%) der Schulen mitgebracht werden, meistens aber nicht (37.8\%) und seltener nur in der Pause $(29.7 \%)$ genutzt werden. Mit der Strenge dieser Regelungen sind die meisten der Lehrkräfte voll (56.4\%) oder zum Teil (25.6\%) zufrieden. ${ }^{218}$

Um einen vertiefenderen Eindruck der Thematisierung von Bildschirmmedien in Klasse und Unterricht zu erhalten, wurden die Lehrkräfte zu allen Messzeitpunkten danach gefragt wie das Thema Medien bisher in der Klasse unterrichtet wurde. ${ }^{219}$ Damit sollte zum einen abgeklärt werden wie viel Medienunterricht bereits vor dem Unterrichtsprogramm des Berliner Längsschnitts Medien in den Klassen durchgeführt wurde und wie dieser verankert war. Zum anderen soll damit aber auch kontrolliert werden, inwiefern in Baseline- und Kontrollklassen „eigenständig“" Medienunterricht durchgeführt wurde, der nicht Teil des Projektes war. Vor Beginn der Längsschnittuntersuchung im November 2005 hatte das Thema Medien nach Auskunft der Lehrkräfte in rund 62 Prozent der Klassen fast keine Rolle gespielt, wobei es noch häufiger in den Interventionsklassen als den Kontrollklassen speziell thematisiert wurde (siehe Tabelle 82). Wurde das Thema Medien behandelt, dann bislang immer als Teil des normalen Unterrichts (69\%). Explizit als solchen ausgewiesenen Medienunterricht in den vorangegangenen Schuljahren gab es allerdings lediglich in rund 18 Prozent der Kontrollklassen, aber nicht in Interventions- oder Baselineklassen. In 18 Prozent aller Klassen wurden zusätzlich auch in der Vergangenheit einzelne Stunden unterrichtet, die nur dem Thema Fernsehen oder Video- und Computerspiele gewidmet waren. In einigen Interventionsklassen gab es zudem bereits mindestens einmal Projekttage in der Klasse, die sich mit Medien, ihren Inhalten und Folgen auseinandergesetzt haben. Bis zum Ende der sechsten Klasse ist in allen Klassen eine deutliche Intensivierung der schulischen Auseinandersetzung mit dem Thema Medien, welche erwartungsgemäß am größten in der Interventionsgruppe ist,

218 Inwieweit stimmen Sie den folgenden Aussagen zu? Ich würde mir an meiner Schule strengere Regeln zur Nutzung privater Mediengeräte wünschen. Abstufungen: „trifft zu“, „trifft eher zu“, „trifft eher nicht zu“, „trifft nicht zu“.

219 Wie wurde das Thema Medien in dieser Klasse unterrichtet?: „Im Rahmen des Projektes Mediennutzung und Schulleistung“, „In den vorangegangenen Schuljahren gab es explizit als solchen ausgewiesenen Medienunterricht“, „Medienunterricht ist bislang immer Teil des normalen Unterrichts gewesen“, „In der Klasse wurden in der Vergangenheit Stunden unterrichtet, die nur dem Thema Fernsehen oder Video- und Computerspiele gewidmet waren“, „Es hat bereits einmal Projekttage in der Klasse gegeben, die sich mit Medien, ihren Inhalten und Folgen auseinandergesetzt haben“, „Bisher hat das Thema Medien im Unterricht fast keine Rolle gespielt“". 
zu beobachten. So sind Bildschirmmedien bis dahin deutlich häufiger als Teil des normalen Unterrichts (87\%) behandelt worden. In rund einem Drittel aller Klassen (35\%), mit 56 Prozent aber vor allem in den Interventionsklassen, wurden zudem bis zum Ende der sechsten Klasse einzelne Stunden unterrichtet, die nur dem Thema Fernsehen oder Video- und Computerspiele gewidmet waren. In 50 Prozent der Interventionsklassen sowie 24 Prozent der Kontrollklassen gab es darüber hinaus bereits mindestens einmal Projekttage in der Klasse, die sich mit

Tabelle 82. Wie wurde bisher das Thema Medien in dieser Klasse unterrichtet?

$\begin{array}{lllll}\mathrm{t} 1 & \mathrm{t} 2 & \mathrm{t} 3 & \mathrm{t} 4 & \mathrm{t} 5\end{array}$

\section{Interventionsgruppe}

Im Rahmen des Projektes

Es gab Medienunterricht

$94.7 \% \quad 94.1 \% \quad 83.3 \%$

Teil des normalen Unterrichts

$66.7 \% \quad 66.7 \% \quad 82.4 \% \quad 78.6 \% \quad 81.2 \%$

Std. für TV-/PC-Spiele

$25.0 \%$

$33.3 \%$

$56.2 \%$

$73.3 \%$

$56.2 \%$

Projekttage gegeben

$11.1 \%$

$6.2 \%$

$33.3 \%$

$26.7 \%$

$50.0 \%$

Thema hat keine Rolle gespielt

$55.6 \%$

$37.5 \%$

$11.1 \%$

$6.7 \%$

$20.0 \%$

\section{Kontrollgruppe}

Im Rahmen des Projektes

Es gab Medienunterricht

Teil des normalen Unterrichts

Std. für TV-/PC-Spiele

Projekttage gegeben

Thema hat keine Rolle gespielt
$17.6 \%$

$58.8 \%$

$12.5 \%$

-

$70.6 \%$
$57.1 \% \quad 26.7 \%$

$15.4 \%$

$52.9 \%$

$73.3 \%$

$21.4 \%$

$86.7 \%$

$22.2 \%$

$28.6 \%$

$28.6 \%$

$11.8 \%$

$38.5 \%$

$35.7 \%$

$37.5 \%$

$43.8 \%$
$25.0 \%$

$37.5 \%$

$88.9 \%$

$11.8 \%$

$23.5 \%$

$16.7 \%$

\section{Baselinegruppe}

Im Rahmen des Projektes

Es gab Medienunterricht

Teil des normalen Unterrichts

$100 \%$

$100 \%$

$100 \%$

Std. für TV-/PC-Spiele $\quad 14.3 \%$

$25.0 \%$

$50.0 \% *$

Projekttage gegeben

Thema hat keine Rolle gespielt

$57.1 \%$

$33.3 \% *$

$100 \% *$

Anmerkung. * Angaben von weniger als 50\% der Lehrkräfte. 
Medien, ihren Inhalten und Folgen auseinandergesetzt haben. Explizit als solchen ausgewiesenen Medienunterricht in den vorangegangenen Schuljahren gab es allerdings wiederum lediglich in rund 69 Prozent der Interventionsklassen, in 38 Prozent der Kontrollklassen und gar nicht in den Baselineklassen. Diese Daten zeigen zum einen, dass sich die Schülerinnen und Schüler der Interventionsklassen während der bisherigen Laufzeit des Berliner Längsschnitt Medien, am intensivsten mit Medien, ihren Chancen und Risiken auseinandergesetzt haben, gefolgt von den Kontrollklassen. In den Baselineklassen waren Bildschirmmedien, wiederum erwartungsgemäß, am seltensten ein Thema. Die Daten zeigen aber auch, dass nicht in allen Interventionsklassen zu allen Befragungszeitpunkten der vorgesehene Medienunterricht durchgeführt wurde (siehe auch Kapitel 7.2.1) und dass auch in einigen Kontrollklassen das laufende Projekt zu unterschiedlichen Zeitpunkten dazu genutzt wurde das Thema Medien im Unterricht zu behandeln.

In den Lehrerfragebögen der sechsten Klasse wurde zusätzlich noch abgefragt, inwieweit die Lehrkräfte ein eigenes Unterrichtsfach Medienunterricht befürworten würden. ${ }^{220}$ Rund die Hälfte der Lehrkräfte (trifft zu: $23.1 \%$, trifft eher zu: $28.2 \%$ ) würden ein derartiges Unterrichtsfach als eher sinnvoll erachten.

\subsubsection{Rückmeldung zum Medienpädagogischen Unterricht}

Bevor auf die Wirksamkeit des durchgeführten Medienunterrichts eingegangen wird, soll in diesem Kapitel dargestellt werden, in welchem Umfang das geplante Unterrichtsprogramm zu den unterschiedlichen Befragungszeitpunkten in den einzelnen Interventionsklassen umgesetzt wurde. Eine ausführliche Konzeptevaluation findet sich bei Kleimann (2011). Wurde das Unterrichtsprogramm durchgeführt, dann in den meisten Fällen vom Klassenlehrer oder der Klassenlehrerin (leider liegen nicht von allen Klassen Rückmeldungen zu dieser Frage vor siehe Tabelle 83).

220 Inwieweit stimmen Sie den folgenden Aussagen zu? Es sollte ein eigenes Fach Medienunterricht geben. Abstufungen: „trifft zu“, „,trifft eher zu“, „trifft eher nicht zu“, „trifft nicht zu“". 


\begin{tabular}{|c|c|c|c|c|}
\hline & \multirow{2}{*}{$\begin{array}{c}\text { Vom } \\
\text { Leicht- } \\
\text { matrosen } \\
\text { zum Me- } \\
\text { dienlot- } \\
\text { sen }\end{array}$} & \multirow[b]{2}{*}{$\begin{array}{c}\text { Die ELI- } \\
\text { ZA-Pro- } \\
\text { tokolle }\end{array}$} & \multicolumn{2}{|c|}{ Medienlotsen gesucht! } \\
\hline & & & $\begin{array}{c}\text { teilge- } \\
\text { nommen } \\
\text { (5 Klas- } \\
\text { sen) }\end{array}$ & $\begin{array}{c}\text { wollten } \\
\text { noch teil- } \\
\text { nehmen } \\
\text { (3 Klassen }\end{array}$ \\
\hline \multicolumn{5}{|l|}{ Durchführung (Klassen) } \\
\hline selbst durchgeführt & 8 & 13 & 3 & 2 \\
\hline mit anderer Person durchgeführt & $1 *$ & 1 & - & - \\
\hline anderer Kollege hat durchgeführt & - & 3 & 2 & - \\
\hline Schüler wollten selbst durchführen & - & - & - & 1 \\
\hline $\begin{array}{l}\text { Anzahl der Stunden des Medienun- } \\
\text { terrichts: } M(S D)\end{array}$ & $14.1(5.3)$ & $11.1(5.3)$ & $11(6.2)$ & 9.5 \\
\hline Median & 7 & 10 & - & - \\
\hline Minimum & 5 & 4 & 5 & - \\
\hline Maximum & 26 & 20 & 20 & - \\
\hline \multicolumn{5}{|l|}{ Fortbildungsveranstaltung } \\
\hline sehr gut & $60.0 \%$ & $33.3 \%$ & - & - \\
\hline eher gut & $33.3 \%$ & $61.1 \%$ & - & - \\
\hline weniger gut & $6.7 \%$ & $5.6 \%$ & - & - \\
\hline \multicolumn{5}{|l|}{ Gestaltung } \\
\hline konkretere Vorschläge erwünscht & $26.3 \%$ & - & - & $1(50 \%)$ \\
\hline Vorschläge genau richtig & $73.7 \%$ & $100 \%$ & $2(50 \%)$ & - \\
\hline gerne freier gewesen & - & - & $2(50 \%)$ & $1(50 \%)$ \\
\hline \multicolumn{5}{|l|}{ Längerfristige Wirkung } \\
\hline auf jeden Fall & $11.1 \%$ & $17.6 \%$ & $1(20 \%)$ & $1(33.3 \%)$ \\
\hline eher ja & $55.6 \%$ & $58.8 \%$ & $3(60 \%)$ & $2(66.6 \%)$ \\
\hline eher nein & $33.3 \%$ & $23.5 \%$ & $1(20 \%)$ & - \\
\hline
\end{tabular}

Anmerkung. Zur ersten und zweiten Intervention liegen Angaben von 19 Lehrkräften vor, zur dritten 5 von 8. Fortbildungsveranstaltung: „Um ihnen die Ziele und Inhalte des Medienunterrichts zu vermitteln, wurden Sie zu einer Fortbildungsveranstaltung eingeladen. Wie gut wurden Sie durch diese auf den Medienunterricht vorbereitet?" (sehr gut, eher gut, weniger gut, gar nicht gut). Gestaltung: „Der Unterricht ist so gestaltet, dass Sie relativ frei in der konkreten Gestaltung sind. Wie beurteilen Sie diesen Aspekt?" (Ich hätte mir mehr konkrete Vorschläge für die Planung und Gestaltung des Unterrichtes gewünscht, Ich wäre in meinen Entscheidungen über Planung und Gestaltung des Unterrichtes gerne freier gewesen, Ich fand die Vorschläge für Planung und Gestaltung des Unterrichtes gerade richtig). Längerfristige Wirkung: Was denken Sie: Hat der Medienunterricht eine längerfristige Wirkung in der Klasse (Ja, auf jeden Fall; eher ja; eher nein; nein, auf keinen Fall).* In einem Fall wurde der Unterricht zum Teil von einem Lehramtsreferendar ausgeführt, der Fragebogen aber von der Lehrkraft ausgefüllt. 
Am Wettbewerb Medienlotsen gesucht! haben sieben Lehrkräfte explizit angegeben nicht teilnehmen zu wollen, zu den anderen Klassen liegen leider wiederum keine Angaben vor. In der dritten Klasse wurden im Durchschnitt 14 Unterrichtstunden, in der vierten und fünften Klasse 11 Unterrichtsstunden für den Medienunterricht verwendet. Die durchgeführten Fortbildungsveranstaltungen wurden von den Lehrkräften fast ausschließlich als eher oder sehr gut bezeichnet. Beim Unterrichtsprogramm Vom Leichtmatrosen zum Medienlotsen hätte sich ein Viertel der Lehrkräfte allerdings noch konkretere Vorschläge zur Umsetzung im Unterricht gewünscht. Beim Unterrichtsprogramm Die ELIZA-Protokolle waren die Lehrkräfte mit der Gestaltung 100-prozentig zufrieden und im Rahmen des Wettbewerbes Medienlotsen gesucht! wären manche Lehrkräfte wiederum gern freier in ihre Gestaltung gewesen. Rund Zweidrittel bis Dreiviertel der befragten Lehrkräfte vermutet auch eher oder auf jeden Fall eine längerfristige Wirkung des jeweiligen Unterrichtsprogramms.

Erste Interventionsphase - Vom Leichtmatrosen zum Medienlotsen. 95 Prozent der Lehrkräfte, die diese Unterrichtsmaßnahme durchgeführt haben, betrachteten diese insgesamt als für das Alter angemessen und gaben an, dass diese auch eher gut oder sehr gut bei den Schülerinnen und Schülern angekommen sei. 74 Prozent beurteilten die Arbeitsblätter als eher oder sehr hilfreich. Tabelle 84 stellt noch einmal dar, zu welchem Ausmaß die unterschiedlichen Komponenten Geschichte, Medientagebuch, 2-Wochenplan, Vertrag, Elternabend und Elternbroschüre von den Klassen angenommen wurden und wie diese hinsichtlich der vier Dimensionen Medienumgang bewusst machen, Folgen des Medienumgangs erkennen, Alternativen entwickeln und Umfeld sensibilisieren zu beurteilen seien ( $\mathrm{Zu}$ den Dimensionen vgl. Kapitel 1.3). ${ }^{221}$ Die Geschichte Vom Leichtmatrosen zum Medienlotsen wurde in 17 Klassen komplett durchgeführt, in 2 Klassen zum Teil und zu einer Klasse liegt keine Rückmeldung vor. Hinsichtlich der Durchführung der einzelnen Kapitel der Geschichte Vom Leichtmatrosen zum Medienlotsen lagen leider nur in zehn Klassen Rückmeldungen vor. Laut diesen Angaben wurden jeweils nur einzelne Kapitel (Wie man hundert Millionen Würmer zählt, Wie man richtig auswertet, Der Zweiwochenplan, Brettspiele und Lerncomputer, Der Vertrag) von jeweils nur einer Klasse nicht behandelt. Der Geschichte wird von den Lehrkräften vor allem auf der Dimension Medienumgang bewusst machen eine Wirkung zugesprochen, etwas weniger auf den beiden Dimensionen Alternativen entwickeln und Folgen erkennen. Die Dimension Umfeld

221 Bitte schätzen Sie die einzelnen Bestandteile des Unterrichts ein. Wie würden sie den Erfolg eines Bestandteiles (Geschichte, Medientagebuch,...) einschätzen und zwar entlang der Dimensionen a) Bewusstmachung des Ausmaßes eigenen Medienkonsums b) des Erkennens und Beurteilens der Folgen des Konsums c) des Aufzeigens von alternativen Freizeitbeschäftigungen d) der Sensibilisierung des Umfeldes. Letztere wurde nur für den Elternabend und die Elternbroschüre abgefragt. 
Tabelle 84. Durchführung und erwartete Wirkung der Schüler- und Elternintervention Vom Leichtmatrosen zum Medienlotsen.

\begin{tabular}{cc|c|c|c|c|}
\hline $\begin{array}{c}\text { Ge- } \\
\text { schichte } \\
\text { Leicht- } \\
\text { matrose }\end{array}$ & $\begin{array}{c}\text { Medien- } \\
\text { tage- } \\
\text { buch }\end{array}$ & $\begin{array}{c}\text { 2-Wo- } \\
\text { chen- } \\
\text { plan }\end{array}$ & $\begin{array}{c}\text { Vertrag } \\
\text { altern- }\end{array}$ & $\begin{array}{c}\text { Eltern- } \\
\text { broschü- } \\
\text { re }\end{array}$ \\
\hline
\end{tabular}

\section{Durchführung (Klassen)}

vollständig

zum Teil

gar nicht

Keine Angabe

als Teil eines

Elternabends

als extra

Elternabend

gar nicht

17

2

$-$

1

\section{Medienumgang bewusst machen}

gering

mittel

hoch

$\begin{array}{cc}- & 6.2 \% \\ 27.8 \% & 31.2 \% \\ 72.8 \% & 62.5 \%\end{array}$

$11.8 \%$

$52.9 \%$

$35.3 \%$

$\begin{array}{ll}11.8 \% & 13.3 \% \\ 41.2 \% & 60.0 \% \\ 47.1 \% & 26.7 \%\end{array}$

$25.0 \%$

$56.2 \%$

$18.8 \%$ hoch

\section{Folgen erkennen}

gering
mittel
hoch

\section{Umfeld sensibilisieren}

gering

mittel

hoch

$\begin{array}{crrr}21.4 \% & 22.2 \% & 6.7 \% & 14.3 \% \\ 71.4 \% & 66.7 \% & 40.0 \% & 50.0 \% \\ 7.1 \% & 11.1 \% & 53.3 \% & 35.7 \%\end{array}$

$35.7 \%$

$22.2 \%$

$26.7 \%$

$28.6 \%$

$42.9 \%$

$55.6 \%$

$33.3 \%$

$50.0 \%$

$21.4 \%$

$22.2 \%$

$40.0 \%$

$21.4 \%$
9

8

2

$13.3 \%$

$46.7 \%$

$40.0 \%$

sensibilisieren wurde nur für den Elternabend und die Elternbroschüre abgefragt. Das Medientagebuch wurde in 16 Klassen komplett und in 2 Klassen zum Teil durchgeführt. Zu weiteren zwei Klassen liegen, wie für die beiden folgenden Komponenten, keine Rückmeldungen vor. Der 2-Wochenplan wurde nur in acht Klassen komplett, in sieben Klassen zum Teil und in drei Klassen gar nicht durchgeführt. Der Vertrag wurde nur noch in drei Klassen komplett, in sieben Klassen zum Teil und in acht Klassen gar nicht durchgeführt. Insgesamt sehen 
die Lehrkräfte die Wirkung der drei Komponenten Medientagebuch, 2-Wochenplan und Vertrag vor allem in der Bewusstmachung des eigenen Medienumgangs. Elternabende wurden in 17 Klassen (in 9 Klassen als extra Abend) durchgeführt, in zwei Klassen fand kein Elternabend statt. Nach Einschätzung der Lehrkräfte haben durchschnittlich 65.2 Prozent $(S D=22,3)$ der Eltern die Elternabende besucht. Wurde der Elternabend mit weiteren organisatorischen oder inhaltlichen Dingen verknüpft, lag die durchschnittliche Teilnahmequote mit 73 Prozent deutlich über der Teilnahmequote bei reinen Themenelternabenden (57\%). Laut Angaben der Lehrkräfte sind die Elternabende auch insbesondere geeignet um den eigenen Medienumgang der Kinder bewusst zu machen. Des Weiteren sensibilisieren diese zu einem geringeren Maß auch das soziale Umfeld der Kinder. Die Elternbroschüre, welche an den Elternabenden verteilt wurde, (die beiden Klassen, welche keine Elternabende durchgeführt hatten, bekamen die Broschüren zugeschickt), wurde schließlich in ihrer Wirkung von den Lehrkräften annähernd identisch auf allen vier Dimensionen beurteilt.

Zweite Interventionsphase - Die ELIZA-Protokolle. 72 Prozent der Lehrkräfte die diese Unterrichtsmaßnahme durchgeführt hatten, betrachteten diese insgesamt als für das Alter angemessen. Die übrigen 28 Prozent waren der Meinung, die behandelte Geschichte sei eher für ältere Kinder geeignet gewesen. Alle Lehrkräfte, die diese Fragen im Lehrerfragebogen beantworteten, gaben zudem an, dass die Geschichte eher gut oder sehr gut bei den Schülerinnen und Schülern angekommen sei und beurteilten die Arbeitsblätter als eher oder sehr hilfreich. Tabelle 85 stellt noch einmal dar wie das Unterrichtsprogramm Die ELIZA-Protokolle wiederum hinsichtlich der vier Dimensionen Medienumgang bewusst machen, Folgen des Medienumgangs erkennen, Alternativen entwickeln und Umfeld sensibilisieren von den Lehrkräften beurteilt wurde und welche Kapitel der Geschichte durchgeführt wurden. ${ }^{222}$ Die Lehrkräfte erwarteten insgesamt von der Geschichte einen Erfolg dahin gehend, dass die Kinder vor allem die Folgen eines exzessiven Medienkonsums erkennen und sich zu einem geringeren Ausmaß auch ihren eigenen Medienumgang bewusst machen und Alternativen zu diesem entwickeln. Von 16 Klassen wurden die sieben Kapitel des Unterrichtsprogramms Die ELIZA-Protokolle in ihrer Gänze bearbeitet, wobei die einzelnen Fragen oder Aufgaben zu den Kapiteln in manchen Klassen nur teilweise oder in seltenen Fällen gar nicht bearbeitet wurden (siehe Tabelle 85). In einer Klasse wurde das Unterrichtsprogramm nur zum Teil bearbeitet, in weiteren zwei Klassen gar nicht.

222 Wie schätzen Sie den Erfolg des Unterrichts im Hinblick auf die folgenden Punkte ein? a) Bewusstmachen des Ausmaßes eigenen Medienkonsums (gering, mittel, hoch), b) Erkennen problematischer Mediennutzung (gering, mittel, hoch), c) Aufzeigen von Alternativen Möglichkeiten der Freizeitgestaltung (gering, mittel, hoch). 
Tabelle 85. Durchführung und erwartete Wirkung der Schülerintervention Die ELIZA-Protokolle.

\begin{tabular}{cccc}
\hline & \multicolumn{3}{c}{ Durchführung (Klassen) } \\
\cline { 2 - 5 } bearbeitet & $\begin{array}{c}\text { teilweise be- } \\
\text { arbeitet }\end{array}$ & $\begin{array}{c}\text { nicht bear- } \\
\text { beitet }\end{array}$ & $\begin{array}{c}\text { keine Anga- } \\
\text { ben }\end{array}$ \\
\hline
\end{tabular}

Gesamt

16

2

1

\section{Nachtschicht}

Kapitel gelesen

Fragen bearbeitet

Aufgaben bearbeitet

2. Gespräch in der Dunkelheit

Kapitel gelesen

Fragen bearbeitet

Aufgaben bearbeitet

8

3. Meister aller Waffen

Kapitel gelesen

Fragen bearbeitet

Aufgaben bearbeitet

8

6

3

\section{Fiona}

Kapitel gelesen

Fragen bearbeitet

Aufgaben bearbeitet

\section{Mario}

Kapitel gelesen

Fragen bearbeitet

Aufgaben bearbeitet

6. Notbremse

Kapitel gelesen

Fragen bearbeitet

-
8
7

\section{Abschied}

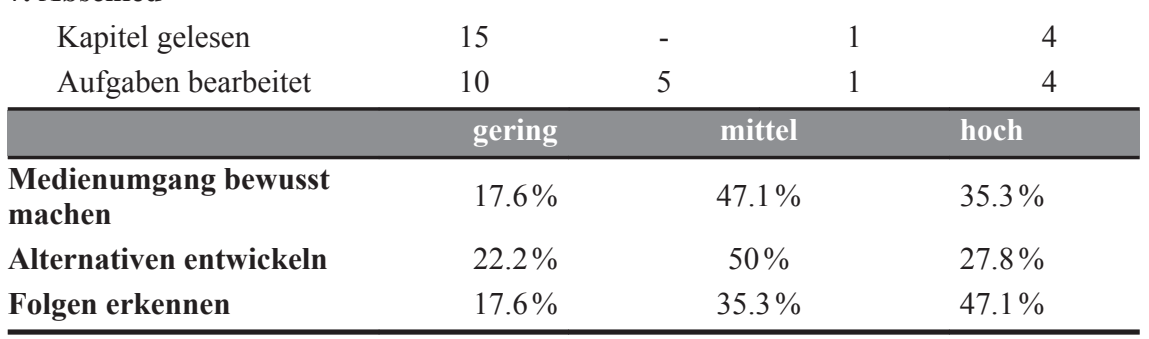


Dritte Interventionsphase - Medienlotsen gesucht! In der fünften Klasse gestaltete es sich aufgrund unterschiedlicher Gründe (siehe Kapitel 1.3.3) am schwierigsten die Lehrkräfte an der Teilnahme am Medienunterrichtsprogramm zu motivieren. Letztlich beteiligten sich nur fünf Klassen an dem ausgeschriebenen Wettbewerb, drei weitere gaben an noch teilnehmen zu wollen. Fünf der Lehrkräfte, die diese Unterrichtsmaßnahme durchgeführt haben, oder planten durchzuführen, betrachteten diese insgesamt als für das Alter angemessen (siehe Tabelle 86). Zwei Lehrkräfte waren der Meinung, der Wettbewerb sei eher für ältere Kinder geeignet gewesen. Alle Lehrkräfte, die diese Fragen im Lehrerfragebogen beantworteten, gaben zudem an, dass der Wettbewerb Medienlotsen gesucht! eher gut oder sehr gut bei den Schülerinnen und Schülern angekommen sei. Insgesamt erwarteten die Lehrkräfte von dem Wettbewerb, dass er den Kindern helfe das Ausmaß ihrer eigenen Mediennutzung und zu einem geringeren Anteil auch die Folgen zu erkennen sowie Alternativen zu entwickeln. ${ }^{223}$

Tabelle 86. Beurteilung und erwartete Wirkung der Schülerintervention Medienlotsen gesucht!.

\section{teilgenommen (5 Klassen)}

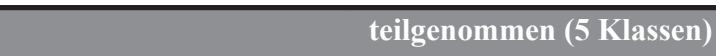

wollen noch teilnehmen

(3 Klassen)

\section{Unterrichtsprojekt}

angemessen

eher für ältere Kinder

sehr gut angekommen

eher gut angekommen

\begin{tabular}{l|l}
2 \\
2 \\
2 \\
2
\end{tabular}

3

1

\begin{tabular}{lccc|ccc}
\hline & gering & mittel & hoch & gering & mittel & hoch \\
\hline $\begin{array}{l}\text { Medienumgang bewusst } \\
\text { machen }\end{array}$ & - & 3 & 2 & - & 2 & 1 \\
Alternativen entwickeln & - & 4 & 1 & - & 2 & 1 \\
Folgen erkennen & - & 4 & 1 & - & 2 & 1 \\
\hline
\end{tabular}

Diese Rückmeldungen zum Medienunterricht verdeutlichen recht anschaulich zu welchem Ausmaß in den Interventionsklassen zu den Befragungszeitpunkten der vorgesehene Medienunterricht durchgeführt wurde. Insgesamt verlief die Umsetzung des medienpädagogischen Unterrichts im Berliner Längsschnitt Medien recht erfolgreich, jedoch mit einem deutlichen Einbruch bei der Durchführung in der fünften Klasse.

223 Wie schätzen Sie den Erfolg des Unterrichts im Hinblick auf die folgenden Punkte ein? a) Bewusstmachen des Ausmaßes eigenen Medienkonsums (gering, mittel, hoch), b) Erkennen problematischer Mediennutzung (gering, mittel, hoch), c) Aufzeigen von Alternativen Möglichkeiten der Freizeitgestaltung (gering, mittel, hoch). 


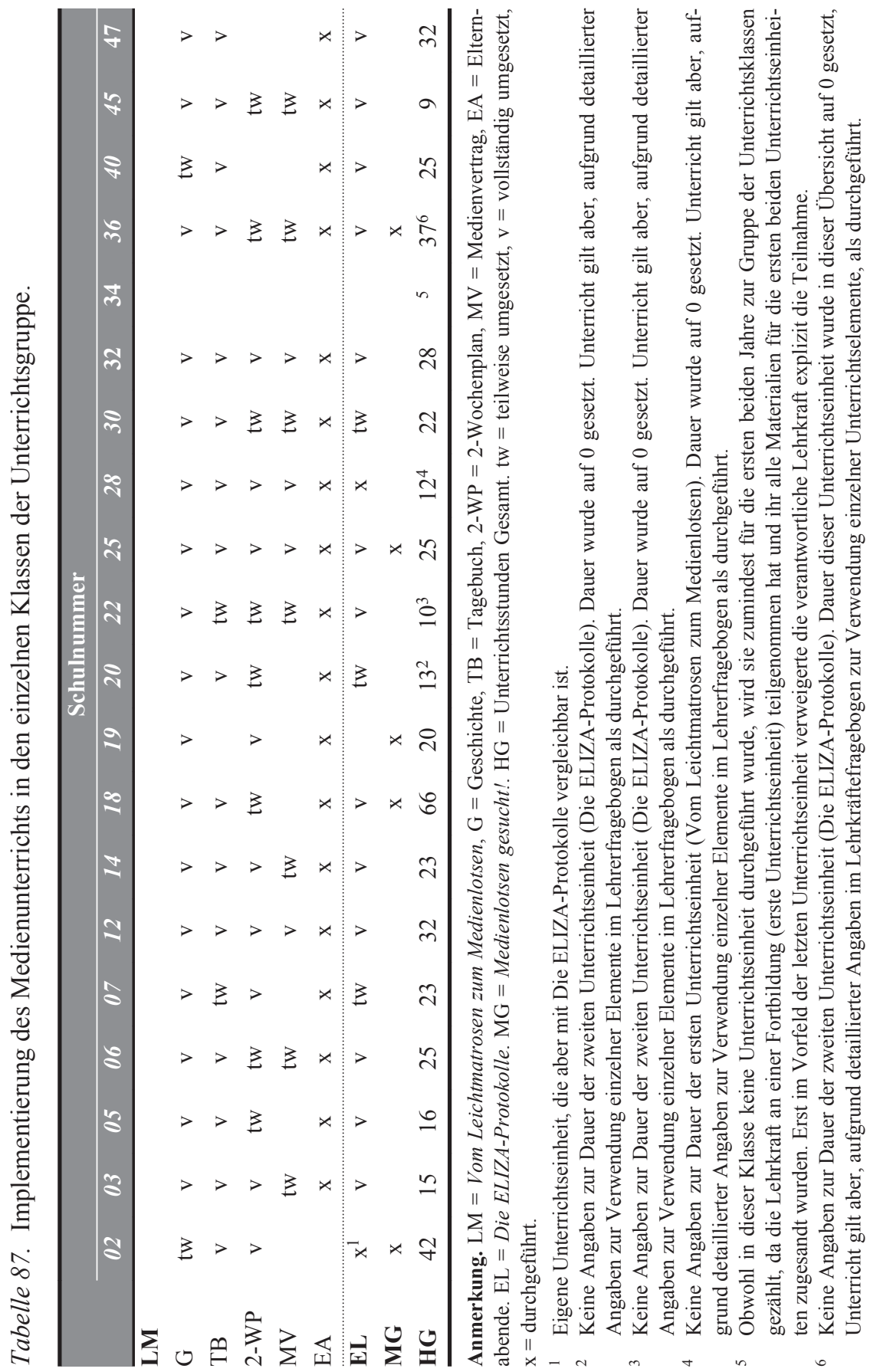


Das Unterrichtskonzept wurde vom Großteil der zufällig ausgewählten Grundschullehrkräfte der Interventionsklassen ab der dritten Klasse im Rahmen des regulären Deutsch- bzw. Sachkundeunterrichts umgesetzt und als tauglich erachtet, auf den Dimensionen Medienumgang bewusst machen, Alternativen entwickeln, Folgen erkennen und (zu geringerem Ausmaß) Umfeld sensibilisieren seine Wirkung entfalten zu können. Allerdings setzten nicht alle ausgewählten Klassen den Medienunterricht um und nicht immer kamen alle Bestandteile des Unterrichtskonzeptes zum Einsatz (Tabelle 87 liefert noch einmal einen Gesamtüberblick zur Implementierung des Medienunterrichts in den einzelnen Schulklassen der Interventionsgruppe). Von den Schülerinnen und Schülern wurde dieses Programm nach Auskunft der Lehrkräfte der Unterrichtsklassen im Großen und Ganzen gut angenommen.

Von besonderem Interesse ist nun die Frage, ob das Medienunterrichtsprogramm auch seine gewünschten Wirkungen hinsichtlich der kindlichen Mediennutzung entfalten konnte und ferner, ob auch bezüglich der oben betrachteten Variablen Schulleistung, Gewaltverhalten, Computerspielabhängigkeit sowie Fettleibigkeit unterschiedliche Verläufe zwischen Interventions- und Kontrollklassen beobachtet werden können. Zur weiteren Evaluation des medienpädagogischen Unterrichtsprogramms wurden nur diejenigen Schülerinnen und Schüler der Ausgangsstichprobe (vgl. Kapitel 1.4.1) in die Analyse aufgenommen, zu denen zu allen Messzeitpunkten Daten vorlagen (Interventions- und Kontrollgruppe: 5 Messzeitpunkte, Baselinegruppe 3 Messzeitpunkte). Die Evaluationsstichprobe reduziert sich somit von 1.207 auf 559 Schülerinnen und Schüler (Interventionsgruppe: $n=249$; Kontrollgruppe: $n=246$; Baselinegruppe: $n=64) .224$

Im Vergleich zur Ausgangsstichprobe sind in der Evaluationsstichprobe keine Abweichungen in der Altersstruktur (auch nicht bei einer nach Gruppen getrennten Betrachtung), geringe Abweichungen in der Geschlechterverteilung (insbesondere ein geringerer Anteil an Jungen in den Interventionsklassen der Evaluationsstichprobe), geringe Abweichungen im Anteil an Kindern mit Migrationshintergrund (insbesondere ein deutlich geringerer Anteil an Kindern mit Migrationshintergrund in den Baselineklassen der Evaluationsstichprobe) und geringe Abweichungen im Anteil an Kindern mit mittlerem und hohen Bildungsniveau (insbesondere ein deutlich geringerer Anteil an Kindern mit hohem Bildungsniveau in den Interventionsklassen der Evaluationsstichprobe) zu beobachten (vgl. Tabelle 88). Somit sind wie in der Ausgangsstichprobe auch in der Evaluationsstichprobe Im Vergleich zur Gesamtstichprobe in der Baselinegruppe deutlich mehr Kinder aus einem Elternhaus mit hohem Bildungsniveau (66.7\%) vertreten. In der Kontrollgruppe der Evaluationsstichprobe liegt der Anteil mit 52 Pro-

224 Diese Beschränkung ermöglicht es am ehesten etwaige Gruppenunterschiede auf die durchgeführte Intervention zurückzuführen. 
Tabelle 88. Stichprobenbeschreibung der Evaluationsstichprobe (Schülerinnen und Schüler nach Untersuchungsgruppe).

\begin{tabular}{|c|c|c|c|c|c|c|c|c|}
\hline & \multicolumn{2}{|c|}{ Gesamt } & \multicolumn{2}{|c|}{ Baselinegruppe } & \multicolumn{2}{|c|}{ Kontrollgruppe } & \multicolumn{2}{|c|}{$\begin{array}{l}\text { Interventions- } \\
\text { gruppe }\end{array}$} \\
\hline & AS & ES & $\mathbf{A S}$ & ES & $\mathbf{A S}$ & ES & AS & ES \\
\hline \multicolumn{9}{|l|}{ Alter } \\
\hline $\begin{array}{l}\text { 3. Klasse } \\
\text { (Halbjahr) }\end{array}$ & $8 ; 11$ & $8 ; 11$ & $8 ; 11$ & $8 ; 11$ & $8 ; 11$ & $8 ; 11$ & $8 ; 11$ & $8 ; 11$ \\
\hline 3. Klasse & $9 ; 6$ & $9 ; 6$ & - & - & $9 ; 6$ & $9 ; 6$ & $9 ; 6$ & $9 ; 6$ \\
\hline 4. Klasse & $10 ; 5$ & $10 ; 5$ & - & - & $10 ; 5$ & $10 ; 5$ & $10 ; 5$ & $10 ; 6$ \\
\hline 5. Klasse & $11 ; 5$ & $11 ; 5$ & $11 ; 5$ & $11 ; 5$ & $11 ; 6$ & $11 ; 5$ & $11 ; 5$ & $11 ; 5$ \\
\hline 6. Klasse & $12 ; 5$ & $12 ; 5$ & $12 ; 6$ & $12 ; 6$ & $12 ; 5$ & $12 ; 5$ & $12 ; 5$ & $12 ; 5$ \\
\hline $\begin{array}{l}\text { Anteil } \\
\text { männlich }\end{array}$ & $51.7 \%$ & $50.4 \%$ & $48.9 \%$ & $50 \%$ & $54.4 \%$ & $53.7 \%$ & $50.0 \%$ & $45.4 \%$ \\
\hline $\begin{array}{l}\text { Anteil Migra- } \\
\text { tionshinter- } \\
\text { grund }\end{array}$ & $27.5 \%$ & $25.6 \%$ & $29.6 \%$ & $17.2 \%$ & $27.4 \%$ & $28.0 \%$ & $27.2 \%$ & $25.3 \%$ \\
\hline \multicolumn{9}{|l|}{$\begin{array}{l}\text { Bildungs- } \\
\text { niveau }\end{array}$} \\
\hline niedrig & $13.1 \%$ & $11.6 \%$ & $9.6 \%$ & $6.7 \%$ & $15.5 \%$ & $11.7 \%$ & $11.9 \%$ & $12.7 \%$ \\
\hline mittel & $33.1 \%$ & $36.0 \%$ & $30.7 \%$ & $26.7 \%$ & $35.7 \%$ & $36.0 \%$ & $31.2 \%$ & $38.4 \%$ \\
\hline hoch & $53.8 \%$ & $52.4 \%$ & $59.6 \%$ & $66.7 \%$ & $48.8 \%$ & $52.3 \%$ & $56.9 \%$ & $48.9 \%$ \\
\hline
\end{tabular}

Anmerkung. AS = Ausgangsstichprobe. $\mathrm{ES}=$ Evaluationsstichprobe.

zent auf dem Niveau der Gesamtstichprobe (in der Ausgangsstichprobe lagen sie noch leicht darunter). In der Interventionsgruppe der Evaluationsstichprobe liegen die Kinder aus einem Elternhaus mit hohem Bildungsniveau mit 49 Prozent leicht unter dem Niveau der Gesamtstichprobe (in der Ausgangsstichprobe lagen sie noch leicht darüber).

\subsubsection{Mediennutzung im Gruppenvergleich}

Eine erste Möglichkeit die Erfolge des durchgeführten Medienunterrichtsprogrammes zu beurteilen, besteht in der Betrachtung der Entwicklung der kindlichen Mediennutzung getrennt für die unterschiedlichen Untersuchungsgruppen hinsichtlich Bildschirmmedienausstattung im Kinderzimmer, Bildschirmmediennutzungszeiten, konsumierter Bildschirmmedieninhalte sowie elterlicher Medienerziehung. 
Bildschirmmedienausstattung. Insgesamt lässt sich eine Zunahme mit eigenen Bildschirmmediengeräten (TV, PC, Spielkonsole und tragbare Spielkonsole) im Kinderzimmer über die Zeit feststellen (siehe Kapitel 2.2.1). Betrachtet man die Untersuchungsgruppen getrennt, so zeigt sich, dass diese Zunahme in der Interventionsgruppe im Vergleich zu den anderen beiden Gruppen bis zum Ende der sechsten Klasse etwas geringer (TV-Geräte, PC - hier jedoch nicht im Vergleich zur Baselinegruppe) oder zumindest etwas verzögert (Spielkonsole) ist. So nahm der Anteil der Kinder mit einem eigenen Fernseher im Kinderzimmer in der Interventionsgruppe nur um neun Prozent zu (vgl. Abbildung 80). In der Kontrollgruppe, die bereits zum ersten Messzeitpunkt besser mit Fernsehgeräten ausgestattet war $\left(\chi^{2}(1, N=476)=0.40, p=.58\right)$, ist jedoch ein Zuwachs von 14 Prozent und in der Baselinegruppe, die in der dritten Klasse geringer als die beiden anderen Gruppen ausgestattet war, ein Zuwachs von 18 Prozent zu verzeichnen. Beim direkten Vergleich von Kontroll- und Interventionsklassen ${ }^{225}$ zeigt sich, dass lediglich für die Kontrollgruppe ein statistisch bedeutsamer stetiger Zuwachs beobachtet werden kann (Cochrans- $Q(4, N=197)=33.04, p<.01)$. In der Interventionsgruppe stagniert die Ausstattung mit Fernsehern zwischen den Messungen der dritten und fünften Klasse, lediglich nach der fünften Klasse ist wiederum ein statistisch bedeutsamer Zuwachs zu verzeichnen (t 1 bis t 5: Cochrans- $Q(4, N=212)=6.89 p=.14$; t 4 bis t 5 : Cochrans $Q(1, N=236)=6.43$ $p<.05)$. $\mathrm{Zu}$ den Messzeitpunkten t $3\left(\chi^{2}(1, N=476)=5.81, p<.05\right)$ und t $4\left(\chi^{2}\right.$ $(1, N=473)=5.29, p<.05)$ unterscheiden sich die Schülerinnen und Schüler der Interventionsgruppe statistisch bedeutsam von denen der Kontrollgruppe. ${ }^{226} \mathrm{Da}$ die beobachteten Gruppenunterschiede zwischen Interventions- und Kontrollklassen statistisch bedeutsam wurden, können zur Beurteilung der Stärke des beobachteten Effektes die beiden Maße Binomial Effect Size Display (BESD), einem für zufällige Erfolge in der Kontrollgruppe kontrollierten Maß für die Erfolgsquote einer Maßnahme (Peck, 1999; Berkowitz, 1990), sowie $d^{*}$, einem Chi-Quadrat-basierten Effektstärkemaß für Vierfeldertafeln (Prasad \& Smith, 1994), herangezogen werden. Grundlage hierfür sollen die Daten von t 3 (4. Klasse), als einem Indikator kurzfristiger Wirkung nach Durchführung der ersten beiden Unterrichtseinheiten, sowie die Daten von 5 (6. Klasse), als einem Indikator der Langzweitwirkung des medienpädagogischen Unterrichts, sein. Ein $B E S D=.11$ zu t 3 weist darauf hin, dass hinsichtlich der Ausstattung mit Fernsehgeräten bei elf Prozent der Schülerinnen und Schüler durch die Intervention ein überzufälliger Effekt erreicht werden konnte. Dies entspricht einer Effektstär-

225 Die Baseline-Klassen wurden aufgrund der deutlich geringeren Stichprobengröße und dem Vorliegen von Daten zu lediglich zwei Messzeitpunkten aus den Signifikanzprüfungen ausgeschlossen.

226 t $2: \chi^{2}(1, N=471)=.28, p=.60 ;$ t $5: \chi^{2}(1, N=486)=3.43, p=.06$. 
ke $d^{*}=.25$ und somit einem schwachen Effekt. Hinsichtlich der langfristigen Wirkung zeigte sich eine Erfolgsquote von $B E S D=.08 \mathrm{bzw} . d^{*}=0.19$, was wiederum einem schwachen Effekt entspricht.

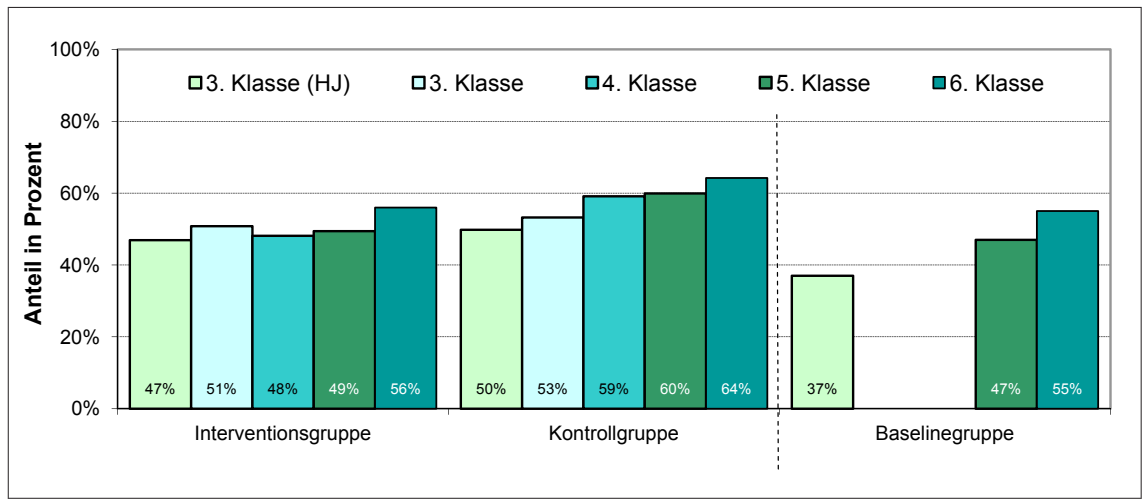

Abbildung 80. Anteil der Kinder mit Fernseher im Kinderzimmer nach Messzeitpunkt und Untersuchungsgruppe. ${ }^{227}$

Geht es um die eigene Spielkonsole im Kinderzimmer, so nehmen die Ausstattungsquoten in Kontroll- und Interventionsklassen im vergleichbaren Ausmaß zu. Die Ausstattung mit einer eigenen Spielkonsole im Kinderzimmer ist am Ende der fünften Klasse in der Interventionsgruppe sogar vorübergehend am höchsten, es konnte aber zumindest durch die Interventionsmaßnahme erreicht werden, dass Spielkonsolen bei einigen Kindern erst zu einem späteren Zeitpunkt ins Kinderzimmer kommen (siehe Abbildung 81). So ist dies ab einem gewissen Alter auch nicht mehr problematisch und wohl kaum zu verhindern. Zumindest steht auch nicht allen Kindern der Interventionsgruppe der eigene Fernseher im Kinderzimmer zur Verfügung (eine Passung, die in den anderen Gruppen größer ist), der eigentlich benötigt wird, um mit der Spielkonsole zu spielen. In der Kontrollgruppe, die wiederum bereits zum ersten Messzeitpunkt besser ausgestattet war $\left(\chi_{2}(1, N=469)=2.02, p=.16\right)$, zeigt sich ein statistisch bedeutsamer stetiger Zuwachs an stationären Spielkonsolen im Kinderzimmer (Cochrans- $Q$ $(4, N=189)=63.99, p<.01)$. In der Interventionsgruppe ist der Verlauf in Form einer Stufe zu beobachten (t 1 bis t 5: Cochrans- $Q(4, N=206)=85.48, p<.01)$. So stagniert die Ausstattung zwischen den Messungen der dritten und vierten Klasse (t 1 bis t 3: Cochrans- $Q(2, N=216)=4.39, p=.11)$, legt deutlich zu

$227 N=559$. Kontrollgruppe: k. A.t1 $=11, \mathrm{k} \cdot \mathrm{A}_{\cdot \mathrm{t} 2}=15, \mathrm{k} \cdot \mathrm{A}_{\cdot \mathrm{t} 3}=11, \mathrm{k} \cdot \mathrm{A}_{\cdot \mathrm{t} 4}=14, \mathrm{k} \cdot \mathrm{A}_{\cdot \mathrm{t} 5}=6$; Interventionsgruppe: $\mathrm{k} \cdot \mathrm{A}_{\cdot \mathrm{t} 1}=8, \mathrm{k} \cdot \mathrm{A}_{\cdot \mathrm{t} 2}=9, \mathrm{k} \cdot \mathrm{A}_{\cdot \mathrm{t} 3}=8, \mathrm{k} \cdot \mathrm{A}_{\cdot \mathrm{t} 4}=8, \mathrm{k} \cdot \mathrm{A}_{\cdot \mathrm{t} 5}=3$; Baselinegruppe: $\mathrm{k} \cdot \mathrm{A}_{\cdot \mathrm{t} 1}=4, \mathrm{k} \cdot \mathrm{A}_{\cdot \mathrm{t} 4}=4, \mathrm{k} \cdot \mathrm{A}_{\cdot \mathrm{t} 5}=4$. 
nach der vierten Klasse (t 3 bis t 5: Cochrans- $Q(1, N=232)=32.01, p<.01)$ und stagniert wiederum in der fünften und sechsten Klasse (t 4 bis t 5: Cochrans$Q(1, N=238)=.18, p=.71)$. $\mathrm{Zu}$ den Messzeitpunkten t $2(\chi 2(1, N=468)=$ $3.67, p<.05)$ und t $3\left(\chi_{2}(1, N=473)=5.45, p<.05\right)$ unterscheiden sich die Schülerinnen und Schüler der Interventionsgruppe statistisch bedeutsam von denen der Kontrollgruppe. ${ }^{228}$ Hinsichtlich einer kurzfristigen Wirkung ist dieser Effekt mit einer Effektstärke $d^{*}=.24$ zu t 3 und einem $B E S D=.11$ mit dem der Fernsehausstattung zu vergleichen und als schwach zu bezeichnen. Gleiches gilt für die Beurteilung einer langfristigen Wirkung $\left(B E S D=.08, d^{*}=0.19\right)$.

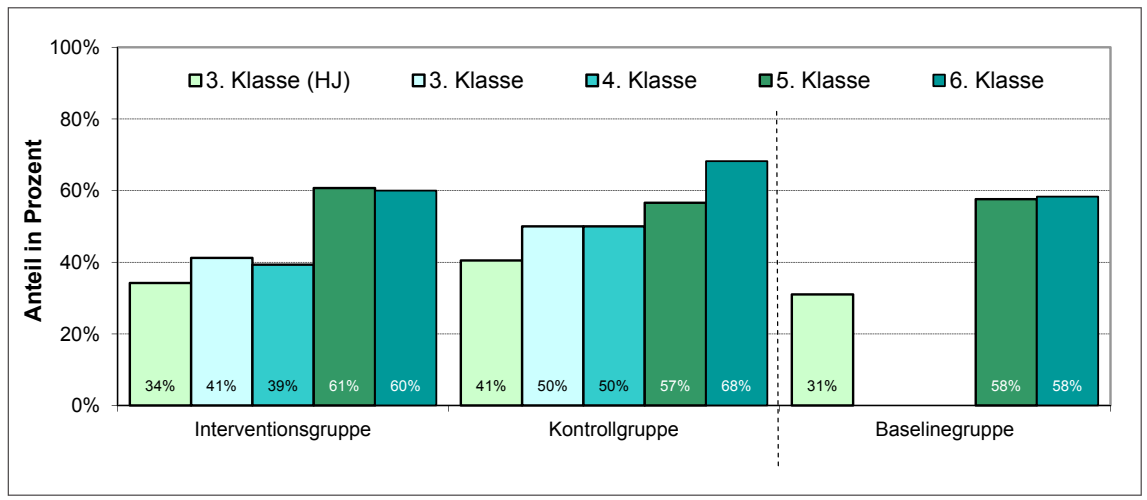

Abbildung 81. Anteil der Kinder mit Spielkonsole im Kinderzimmer nach Messzeitpunkt und Untersuchungsgruppe. ${ }^{229}$

Die deutlichsten Ausstattungsunterschiede zwischen den Gruppen sind für die Ausstattung mit Computern im Kinderzimmer zu beobachten. So nahm der Anteil der Kinder mit einem eigenen PC im Kinderzimmer in der Interventionsgruppe von t 1 zu t 5 um 19 Prozent zu (vgl. Abbildung 82). In der Kontrollgruppe, die im Gegensatz zum Spielkonsolen- und Fernsehgerätebesitz diesmal zum ersten Messzeitpunkt schlechter ausgestattet $\operatorname{war}(\chi 2(1, N=462)=0.44, p=.51)$, ist jedoch ein Zuwachs von 30 Prozent zu verzeichnen.

228 t 4: $\chi^{2}(1, N=468)=.81, p=.37 ; \mathrm{t} 5: \chi^{2}(1, N=487)=3.54, p=.06$.

$229 N=559$. Kontrollgruppe: k. A.t1 $=14$, k. A.t2 $=16, \mathrm{k} \cdot \mathrm{A}_{\cdot \mathrm{t} 3}=12, \mathrm{k} \cdot \mathrm{A}_{\cdot \mathrm{t} 4}=20, \mathrm{k} \cdot \mathrm{A}_{\cdot \mathrm{t} 5}=4$; Interventionsgruppe: $\mathrm{k} . \mathrm{A}_{\cdot \mathrm{t} 1}=12, \mathrm{k} \cdot \mathrm{A}_{\cdot \mathrm{t} 2}=11, \mathrm{k} \cdot \mathrm{A}_{\cdot \mathrm{t} 3}=10, \mathrm{k} \cdot \mathrm{A}_{\cdot \mathrm{t} 4}=7, \mathrm{k} \cdot \mathrm{A}_{\cdot \mathrm{t} 5}=4$ Baselinegruppe: $\mathrm{k} \cdot \mathrm{A}_{\cdot \mathrm{t} 1}=6, \mathrm{k} \cdot \mathrm{A}_{\cdot \mathrm{t} 4}=5, \mathrm{k} \cdot \mathrm{A}_{\cdot \mathrm{t} 5}=4$. 


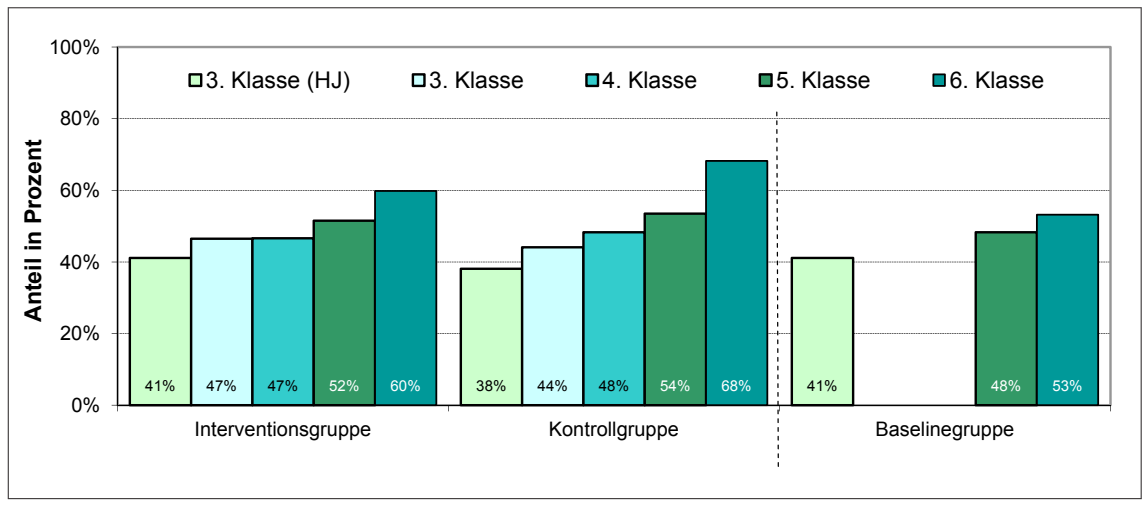

Abbildung 82. Anteil der Kinder mit Computer im Kinderzimmer nach Messzeitpunkt und Untersuchungsgruppe. ${ }^{230}$

In der Kontrollgruppe zeigt sich insgesamt ein statistisch bedeutsamer stetiger Zuwachs an Computern im Kinderzimmer (Cochrans- $Q(4, N=184)=68.31, p$ $<.01)$. In der Interventionsgruppe ist wiederum ein gestaffelter Verlauf zu beobachten (t 1 bis t 5: Cochrans- $Q(4, N=201)=26.92, p<.01)$. So stagniert die Ausstattung mit Computern im Kinderzimmer zwischen den Messungen der dritten und vierten Klasse (t 1 bis t 3: Cochrans- $Q(2, N=211)=2.43 p=.29)$. Nach der vierten Klasse ist wiederum ein statistisch bedeutsamer Zuwachs zu verzeichnen (t 3 bis t 5: Cochrans- $Q(2, N=223)=19.25 p<.05)$. Lediglich zu Messzeitpunkt t 5 unterscheiden sich die Schülerinnen und Schüler der Interventionsgruppe statistisch bedeutsam von denen der Kontrollgruppe $(\chi 2(1, N=485)$ $=3.65, p<.05) .{ }^{231} \mathrm{Da}$ zu t 3 deskriptiv keine Gruppenunterschiede beobachtet werden konnten, wurde auch keine Effektstärke für die kurzfristige Wirkung des durchgeführten Medienunterrichts berechnet. Ein $B E S P=.08$ zu t 5 weist darauf hin, dass langfristig hinsichtlich der Ausstattung mit Computern im Kinderzimmer bei neun Prozent der Schülerinnen und Schüler ein überzufälliger Effekt erreicht werden konnte. Dies entspricht einer Effektstärke $d^{*}=.20$ und somit einem schwachen Effekt.

Eine nachfolgende getrennte Überprüfung auf Treatmentsensitivität der Medienausstattung (TV, Spielkonsole, PC) erbrachte keine bedeutsamen Interaktionen zwischen Bildungsniveau im Elternhaus bzw. Geschlecht der Kinder und einer Untersuchungsgruppenzugehörigkeit.

$230 N=559$. Kontrollgruppe: k. A.t1 $=15$, k. A $\cdot_{\cdot 2}=17, \mathrm{k} \cdot \mathrm{A}_{\cdot \mathrm{t} 3}=14, \mathrm{k} \cdot \mathrm{A}_{\cdot \mathrm{t} 4}=18, \mathrm{k} \cdot \mathrm{A}_{\cdot \mathrm{t} 5}=7$; Interventionsgruppe: $\mathrm{k} \cdot \mathrm{A}_{\cdot \mathrm{t} 1}=18, \mathrm{k} \cdot \mathrm{A}_{\cdot \mathrm{t} 2}=8, \mathrm{k} \cdot \mathrm{A}_{\cdot \mathrm{t} 3}=15, \mathrm{k} \cdot \mathrm{A}_{\cdot \mathrm{t} 4}=10, \mathrm{k} \cdot \mathrm{A}_{\cdot \mathrm{t} 5}=3$; Baselinegruppe: $\mathrm{k}$. A.t1 $=8, \mathrm{k} . \mathrm{A}_{\cdot \mathrm{t} 4}=6, \mathrm{k} \cdot \mathrm{A}_{\cdot \mathrm{t} 5}=2$.

231 t $2: \chi^{2}(1, N=470)=.27, p=.61 ; \mathrm{t} 3: \chi^{2}(1, N=466)=.13, p=.71 ; \mathrm{t} 4: \chi^{2}(1, N=467)=$. $20, p=.66$. 
Bildschirmmediennutzungszeiten. Auch hinsichtlich der Mediennutzungszeiten lässt sich insgesamt eine Zunahme mit dem Alter feststellen (siehe Kapitel 2.2.2). Beim Vergleich der Untersuchungsgruppen zeigt sich deskriptiv zwar, wie oben für die Ausstattung mit Mediengeräten beschrieben, eine Stagnation der mittleren Fernsehnutzungs- und Computerspielzeiten in den Interventionsklassen sowie eine tendenziell stärkere Zunahme dieser von t 1 zu t 5 in den Kontrollklassen. Ebenso liegen die Fernsehnutzungs- und Computerspielzeiten der Schülerinnen und Schüler der Interventionsklassen, bei gleicher oder höherer Ausgangsbasis zu t 1, zu t 3 und zu t 5 unter den Zeiten der Schülerinnen und Schüler der Kontrollklassen (siehe Abbildung 83 und Abbildung 84). Diese Unterschiede manifestieren sich jedoch nicht in einer statistisch bedeutsamen Interaktion zwischen Gruppenzugehörigkeit und Messzeitpunkt (Multivariate (TV, Computerspiele) Varianzanalyse mit Messwiederholung, 5 (Messzeitpunkt) x 2 (Gruppe) $\times 3$ (Bildungsniveau) x 2 (Geschlecht): Messzeitpunkt $F$ $(7 / 299)=6.54, p<.01, f=.42$; Bildungsniveau $F(4 / 612)=3.67, p<.01, f=.15$; Gruppe $F(2 / 305)=3.56, p<.05, f=.15$; Geschlecht $F(2 / 305)=14.73, p<.01$, $f=.29$; keine weiteren bedeutsamen Interaktionen).

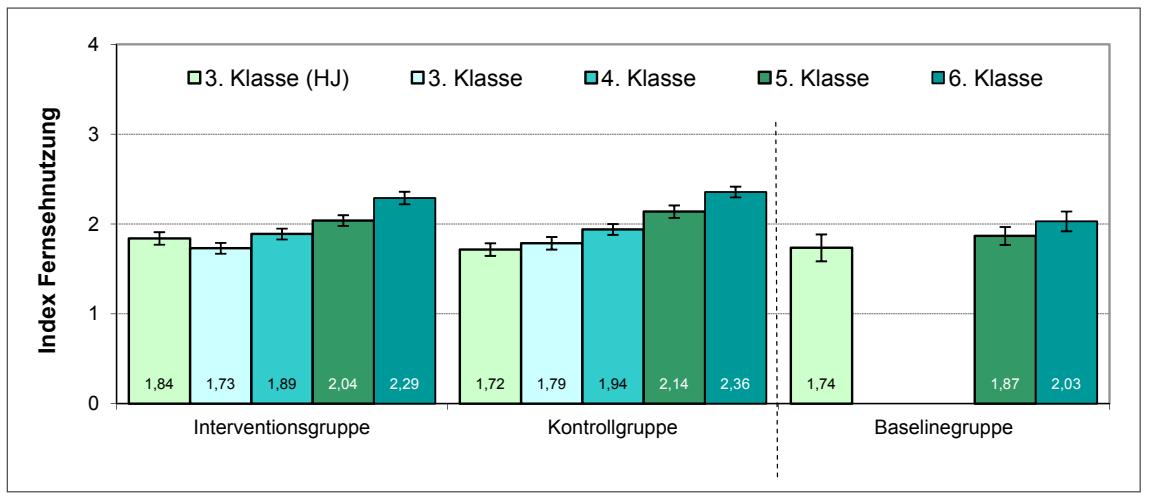

Abbildung 83. Fernsehnutzungszeiten (Index Fernsehnutzung) nach Messzeitpunkt und Untersuchungsgruppe. ${ }^{232}$

$232 N=559$. Kontrollgruppe: k. A.t1 $=16, \mathrm{k} \cdot \mathrm{A}_{\cdot \mathrm{t} 2}=21, \mathrm{k} \cdot \mathrm{A}_{\cdot \mathrm{t} 3}=14, \mathrm{k} \cdot \mathrm{A}_{\cdot \mathrm{t} 4}=13 ; \mathrm{k} \cdot \mathrm{A}_{\cdot \mathrm{t} 5}=9$; Interventionsgruppe: $\mathrm{k} . \mathrm{A}_{\cdot \mathrm{t} 1}=24, \mathrm{k} \cdot \mathrm{A}_{\cdot \mathrm{t} 2}=13, \mathrm{k} \cdot \mathrm{A}_{\cdot \mathrm{t} 3}=14, \mathrm{k} \cdot \mathrm{A}_{\cdot \mathrm{t} 4}=4, \mathrm{k} \cdot \mathrm{A}_{\cdot \mathrm{t} 5}=4$; Baselinegruppe: $\mathrm{k} \cdot \mathrm{A}_{\cdot \mathrm{t} 1}=23, \mathrm{k} \cdot \mathrm{A}_{\cdot \mathrm{t} 4}=3, \mathrm{k} \cdot \mathrm{A}_{\cdot \mathrm{t} 5}=2$. 


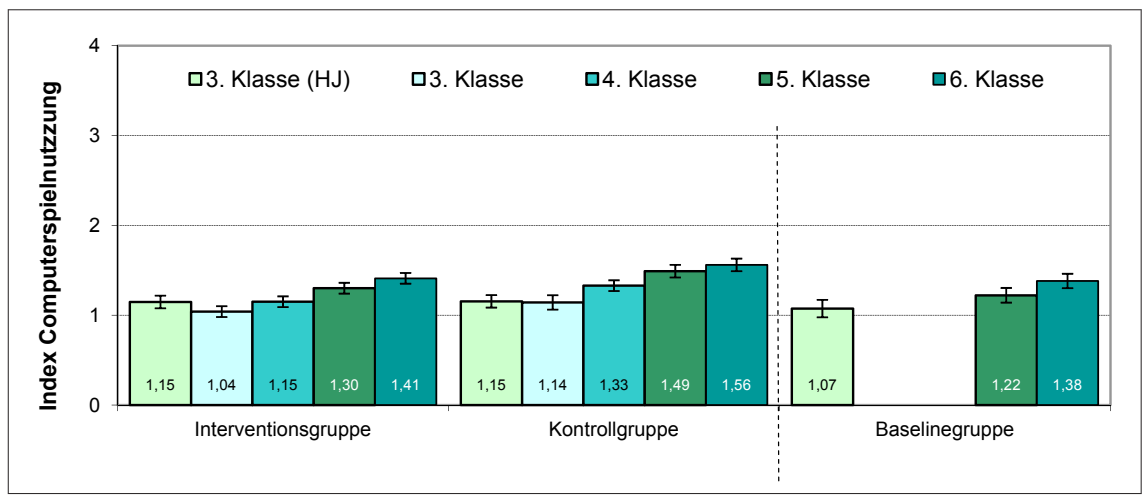

Abbildung 84. Computerspielzeiten (Index Computerspielnutzung) nach Messzeitpunkt und Untersuchungsgruppe. ${ }^{233}$

Bildschirmmedieninhalte. Ein bewussterer Umgang der Schülerinnen und Schüler der Interventionsklassen, der sich in den Spiel- und Fernsehzeiten nur angedeutet hat, zeigt sich deutlicher wenn man die unterschiedlichen Untersuchungsgruppen über den Befragungszeitraum hinsichtlich der konsumierten Bildschirmmedieninhalte vergleicht. So lässt sich zwar erwartungsgemäß in allen Klassen auch eine Zunahme des Konsums von Bildschirminhalten, die erst ab einem Alter von 16 oder 18 Jahren freigegeben sind, bis zum Ende der sechsten Klasse feststellen (siehe Kapitel 2.2.4). Vergleicht man aber die Entwicklung getrennt in den Untersuchungsgruppen, so zeigt sich, dass diese Zunahme des Konsums altersinadäquater Medieninhalte in der Interventionsgruppe bis zum Ende der sechsten Klasse deutlich geringer (Computerspiele) oder zumindest, analog zum Besitz von Spielkonsolen, etwas verzögert (Fernsehen) ist. Beispielsweise nahm der Anteil der Kinder, die schon einmal Filme ab 16 oder 18 Jahren geschaut haben, in allen Untersuchungsgruppen in vergleichbarem Maß von der dritten bis zur sechsten Klasse zu, wobei in der Interventionsgruppe bereits zu Beginn weniger Kinder angaben, solche Inhalte zu schauen $\left(\chi_{2}(1, N=480)=\right.$ $0.29, p=.58)$. Die größte prozentuale Zunahme findet in Letzterer erst in der sechsten Klasse und somit leicht verzögert statt (siehe Abbildung 85). In der Kontrollgruppe zeigt sich insgesamt ein statistisch bedeutsamer stetiger Zuwachs an Kindern, die schon einmal Filme ab 16 oder 18 geschaut haben (Cochrans- $Q$ $(4, N=200)=38.09, p<.01)$. In der Interventionsgruppe stagniert der Konsum derartiger Inhalte zwischen den Messungen der dritten und vierten Klasse ( 1 bis

$233 N=559$. Kontrollgruppe: k. A. $\cdot_{t 1}=30, \mathrm{k} \cdot \mathrm{A}_{\cdot \mathrm{t} 2}=23, \mathrm{k} \cdot \mathrm{A}_{\cdot \mathrm{t} 3}=10, \mathrm{k} \cdot \mathrm{A}_{\cdot \mathrm{t} 4}=11 ; \mathrm{k} \cdot \mathrm{A}_{\cdot \mathrm{t} 5}=2$; Interventionsgruppe: $\mathrm{k} . \mathrm{A}_{\cdot \mathrm{t} 1}=21, \mathrm{k} \cdot \mathrm{A}_{\cdot \mathrm{t} 2}=11, \mathrm{k} \cdot \mathrm{A}_{\cdot \mathrm{t} 3}=12, \mathrm{k} \cdot \mathrm{A}_{\cdot \mathrm{t} 4}=5, \mathrm{k} \cdot \mathrm{A}_{\cdot \mathrm{t} 5}=1$; Baselinegruppe: $\mathrm{k} \cdot \mathrm{A}_{\cdot \mathrm{t} 1}=25, \mathrm{k} \cdot \mathrm{A}_{\cdot \mathrm{t} 4}=2, \mathrm{k} \cdot \mathrm{A}_{\cdot \mathrm{t} 5}=1$. 
t 3: Cochrans- $Q(2, N=225)=1.46 p=.48)$. Nach der vierten Klasse ist wiederum ein statistisch bedeutsamer Zuwachs zu verzeichnen ( 3 bis t 5: Cochrans- $Q$ $(2, N=230)=52.17 p<.01)$. Lediglich zu Messzeitpunkt t 3 unterscheiden sich die Schülerinnen und Schüler der Interventionsgruppe statistisch bedeutsam von denen der Kontrollgruppe $(\chi 2(1, N=473)=8.93, p<.01) .{ }^{234}$ Hinsichtlich einer kurzfristigen Wirkung ist dieser Effekt mit einer Effektstärke $d^{*}=.31$ zu t 3 und einem $B E S D=.14$ als schwach zu bezeichnen. Langfristige Effekte zeigten sich bei der Nutzung altersinadäquater Filminhalte dagegen nicht.

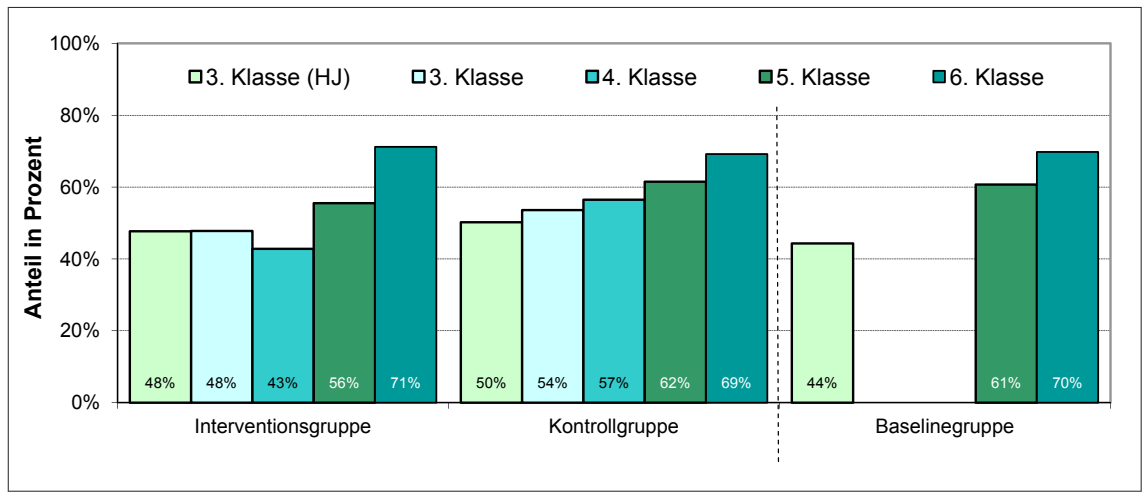

Abbildung 85. Nutzung altersinadäquater Medien (Fernsehen) nach Messzeitpunkt und Untersuchungsgruppe. ${ }^{235}$

Des Weiteren steigt der Anteil der Kinder, die schon einmal Computerspiele ab 16 oder 18 Jahren gespielt haben, in der Interventionsgruppe nur um sieben Prozent. In der Kontroll- $\left(\chi^{2}(1, N=463)=0.32, p=.58\right)$ bzw. der Baselinegruppe, in denen zum ersten Messzeitpunkt vergleichbar oft von den Kindern angegeben wurde Spiele ab 16 oder 18 Jahren gespielt zu haben ist jedoch ein Zuwachs von elf bzw. siebzehn Prozent zu verzeichnen (siehe Abbildung 86).

234 t $2: \chi^{2}(1, N=478)=1.66, p=.19 ; \mathrm{t} 4: \chi^{2}(1, N=479)=1.79, p=.18 ; \mathrm{t} 5: \chi^{2}(1, N=487)$ $=.24, p=.63$.

235 „Hast du dir schon einmal Filme angeschaut, die erst ,ab 16/18' waren?“. $N=559$. Kontrollgruppe: $\mathrm{k} \cdot \mathrm{A}_{\cdot \mathrm{t} 1}=7, \mathrm{k} \cdot \mathrm{A}_{\cdot \mathrm{t} 2}=13, \mathrm{k} \cdot \mathrm{A}_{\cdot \mathrm{t}}=9, \mathrm{k} \cdot \mathrm{A}_{\cdot 4}=12, \mathrm{k} \cdot \mathrm{A}_{\cdot t 5}=6$; Interventionsgruppe: $\mathrm{k} \cdot \mathrm{A}_{\cdot \mathrm{t} 1}=8, \mathrm{k} \cdot \mathrm{A}_{\cdot \mathrm{t} 2}=4, \mathrm{k} \cdot \mathrm{A}_{\cdot \mathrm{t} 3}=13, \mathrm{k} \cdot \mathrm{A}_{\cdot 4}=4 ; \mathrm{k} \cdot \mathrm{A}_{\cdot \mathrm{t} 5}=2$; Baselinegruppe: $\mathrm{k} \cdot \mathrm{A}_{\cdot \mathrm{t} 1}=3, \mathrm{k} \cdot \mathrm{A}_{\cdot \mathrm{t} 4}=3, \mathrm{k} \cdot \mathrm{A}_{\cdot \mathrm{t} 5}=1$. 


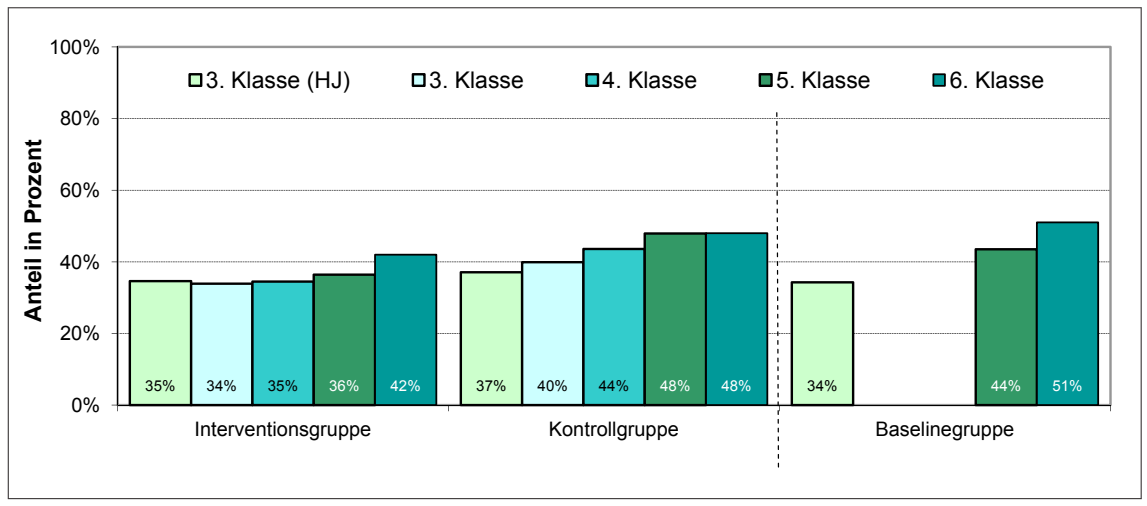

Abbildung 86. Nutzung altersinadäquater Medien (Computerspiele) nach Messzeitpunkt und Untersuchungsgruppe. ${ }^{236}$

Beim direkten Vergleich von Kontroll- und Interventionsklassen zeigt sich, dass lediglich für die Kontrollgruppe ein statistisch bedeutsamer stetiger $\mathrm{Zu}$ wachs beobachtet werden kann (Cochrans- $Q(4, N=192)=11.35, p<.05)$. In der Interventionsgruppe stagniert der Konsum altersinadäquater Computerspiele zwischen der dritten und fünften Klasse bei ca. 35 Prozent und nimmt dann leicht auf 42 Prozent zu (Cochrans- $Q(4, N=207)=7.37, p=.12)$. Lediglich zu den Messzeitpunkten t $3\left(\chi_{2}(1, N=472)=4.14, p<.05\right)$ und t $4\left(\chi_{2}(1, N=478)\right.$ $=6.50, p<.05)$ unterscheiden sich die Schülerinnen und Schüler der Interventionsgruppe statistisch bedeutsam von denen der Kontrollgruppe. ${ }^{237}$ Ein BESD =. 09 zu t 3 weist darauf hin, dass hinsichtlich problematischer Computerspielnutzung bei neun Prozent der Schülerinnen und Schüler ein überzufälliger Effekt erreicht werden konnte. Dies entspricht einer Effektstärke $d^{*}=.21$ und somit einem schwachen Effekt. Hinsichtlich der langfristigen Wirkung zeigte sich keine bedeutsame Erfolgsquote.

Da sich die beiden Geschlechter hinsichtlich der Nutzung altersinadäquater Medieninhalte deutlich voneinander unterscheiden (vgl. Kapitel 2.2.3 und Kapitel 2.2.5, siehe Abbildung 39 und Abbildung 47), wurden zur Untersuchung einer nach Geschlecht unterschiedlichen Treatmentsensitivität obige Analysen getrennt für Jungen und Mädchen berechnet (vgl. Abbildung 87). Unter den Jungen nahm der Anteil derer, die schon einmal Filme ab 16 oder 18 Jahren geschaut haben, in beiden Untersuchungsgruppen von der dritten bis zur sechsten Klasse

236 „Hast du schon einmal Computerspiele gespielt, die erst ,ab 16/18' waren?“. $N=559$. Kontrollgruppe: $\mathrm{k} \cdot \mathrm{A}_{\cdot \mathrm{t} 1}=17, \mathrm{k} \cdot \mathrm{A}_{\cdot \mathrm{t} 2}=18, \mathrm{k} \cdot \mathrm{A}_{\cdot \mathrm{t} 3}=12, \mathrm{k} \cdot \mathrm{A}_{\cdot \mathrm{t} 4}=10, \mathrm{k} \cdot \mathrm{A}_{\cdot \mathrm{t} 5}=10$; Interventionsgruppe: $\mathrm{k} . \mathrm{A}_{\cdot \mathrm{t} 1}=15, \mathrm{k} \cdot \mathrm{A}_{\cdot \mathrm{t} 2}=10, \mathrm{k} \cdot \mathrm{A}_{\cdot \mathrm{t} 3}=11, \mathrm{k} \cdot \mathrm{A}_{\cdot \mathrm{t} 4}=7 ; \mathrm{k} \cdot \mathrm{A}_{\cdot t 5}=16$; Baselinegruppe: $\mathrm{k} . \mathrm{A}_{\cdot+1}=29, \mathrm{k} \cdot \mathrm{A}_{\cdot+4}=2, \mathrm{k} . \mathrm{A}_{\cdot+5}=4$.

237 t $2: \chi^{2}(1, N=467)=1.82, p=.18 ;$ t $5: \chi^{2}(1, N=490)=1.83, p=.18$. 
zu (Intervention: Cochrans- $Q(4, N=98)=23.92, p<.01$; Kontrolle: Cochrans$Q(4, N=106)=35.77, p<.01)$. Für die Mädchen ist lediglich in der Interventionsgruppe eine statistisch bedeutsame Zunahme zu beobachten (Intervention: Cochrans $-Q(4, N=121)=38.75, p<.01$; Kontrolle: Cochrans- $Q(4, N=$ $94)=7.77, p=.10)$. Jungen der Interventionsgruppe unterscheiden sich zudem zu t 3 und t 5 statistisch bedeutsam von denen der Kontrollgruppe t 3: $\chi^{2}(1, N=$ $234)=6.39, p<.05$; t5: $\chi 2(1, N=241)=4.60, p<.01)$. ${ }^{238}$ Für die Mädchen sind keine derartigen Unterschiede zu beobachten, ${ }^{239}$ in der sechsten Klasse liegen die Häufigkeiten in der Interventionsgruppe sogar über denen der Kontrollgruppe. Somit reagieren die Jungen hinsichtlich des Konsums altersinadäquater Filminhalte deutlich besser auf den durchgeführten medienpädagogischen Unterricht als die Mädchen (kurzfristige Wirkung t 3: Jungen $-d^{*}=.38, B E S D=.17$; Mädchen $-d^{*}=.23, B E S D=.08$; langfristige Wirkung t 5: Jungen $-d^{*}=.41, B E S D=.15$ ).

Hinsichtlich der Nutzung von Computerspielen ab 16 oder 18 Jahren nahm unter den Jungen in beiden Untersuchungsgruppen der Anteil derer, die diese schon einmal genutzt haben, von der dritten bis zur sechsten Klasse zu (Intervention: Cochrans- $Q(4, N=91)=11.43, p<.05$; Kontrolle: Cochrans- $Q(4, N=$ $105)=26.27, p<.01$ ). Für die Mädchen, deren Nutzungsniveau von altersinadäquaten Computerspielen sich insgesamt auf einem niedrigen Niveau bewegt, ist weder in der Kontroll- noch in der Interventionsgruppe eine statistisch bedeutsame Veränderung über die Zeit zu beobachten (Intervention: Cochrans- $Q(4, N=$ $116)=1.72, p=.79$; Kontrolle: Cochrans- $Q(4, N=87)=3.91, p=.42)$. Deskriptiv beobachtbare Unterschiede zwischen den Untersuchungsgruppen zu den unterschiedlichen Messzeitpunkten erreichen weder für die Mädchen noch für die Jungen ein statistisch bedeutsames Niveau. ${ }^{240}$ Somit konnten hinsichtlich des Konsums altersinadäquater Computerspielinhalte keine statistisch bedeutsamen Unterschiede in der Wirkung des medienpädagogischen Unterrichts zwischen Jungen und Mädchen festgestellt werden.

238 t 1: $\chi^{2}(1, N=237)=.28 p=.59 ; \mathrm{t} 2: \chi^{2}(1, N=234)=.56, p=.45 ; \mathrm{t} 4: \chi^{2}(1, N=237)=$ $1.80, p=.18$.

239 t 1: $\chi^{2}(1, N=243)=.04, p=.84 ; \mathrm{t} 2: \chi^{2}(1, N=244)=.41, p=.52$; $\mathrm{t} 3: \chi^{2}(1, N=239)=$ $1.54, p=.21 ; \mathrm{t} 4: \chi^{2}(1, N=242)=.00, p=.99 ; \mathrm{t} 5: \chi^{2}(1, N=246)=2.51, p=.11$.

240 Jungen $-\mathrm{t} 1: \chi^{2}(1, N=234)=.30 p=.59 ; \mathrm{t} 2: \chi^{2}(1, N=226)=.08, p=.77 ; \mathrm{t} 3: \chi^{2}(1, N=$ $233)=.24, p=.62 ; \mathrm{t} 4: \chi^{2}(1, N=236)=1.22, p=.27 ; \mathrm{t} 5: \chi^{2}(1, N=242)=.27, p=.61$.

Mädchen $-\mathrm{t} 1: \chi^{2}(1, N=229)=.37 p=.54 ; \mathrm{t} 2: \chi^{2}(1, N=241)=.74, p=.39 ; \mathrm{t} 3: \chi^{2}(1, N$ $=234)=2.73, p=.09 ; \mathrm{t} 4: \chi^{2}(1, N=242)=2.45, p=.12 ; \mathrm{t} 5: \chi^{2}(1, N=248)=.01, p=.98$. 


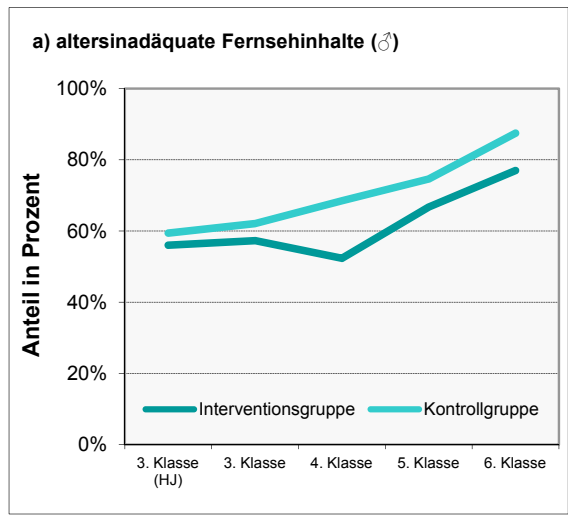

b) altersinadäquate Fernsehinhalte $(+)$
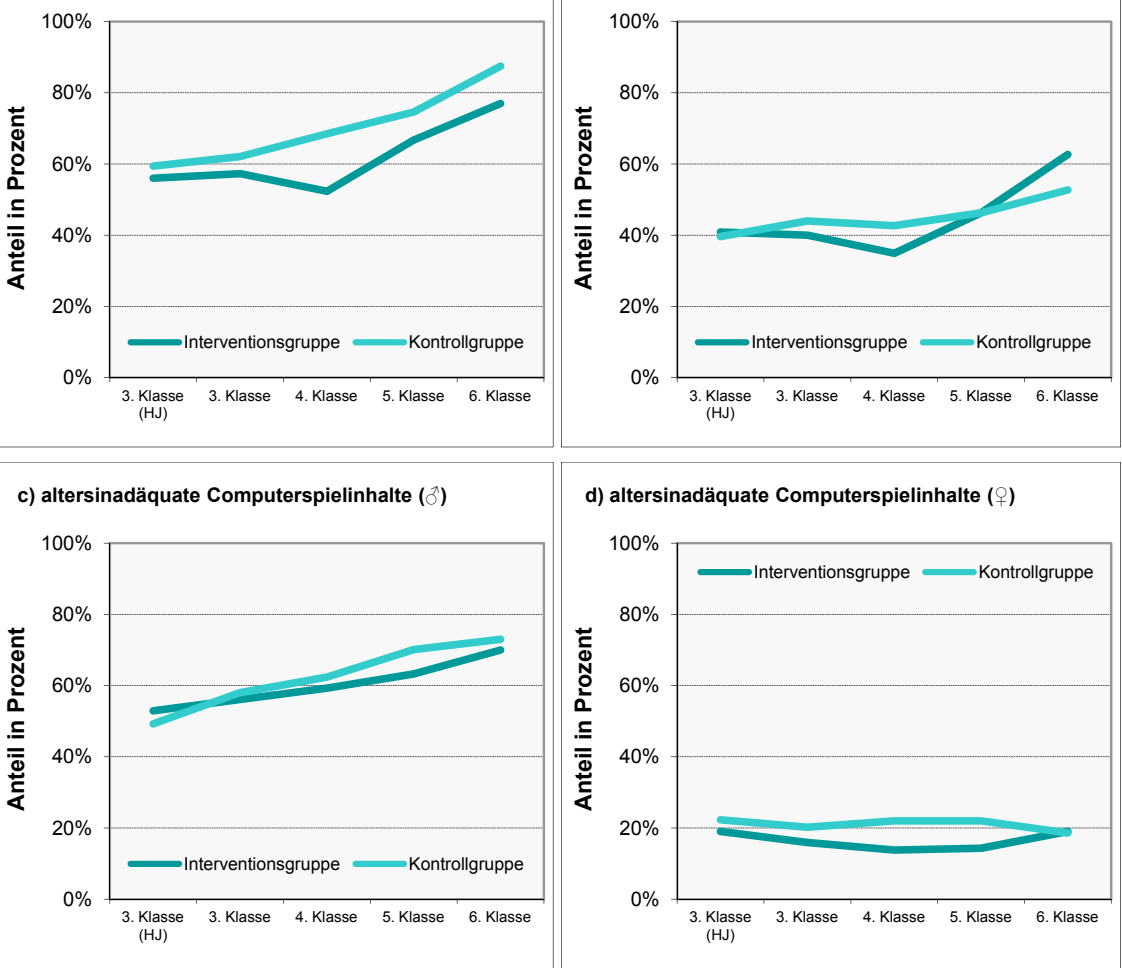

Abbildung 87. Nutzung altersinadäquater Medien (Fernsehen/Computerspiele) nach Geschlecht, Messzeitpunkt und Untersuchungsgruppe.

Medienerziehung. In Kapitel 2.2.4 konnte gezeigt werden, dass sich die Medienerziehung im Elternhaus mit den Bereichen inhaltliche Regeln, Monitoring (d. h. elterliches Interesse für das Medienverhalten der Kinder) und zeitliche Regeln, für das Fernsehen von der dritten bis zur sechsten Klasse nur bedingt ändert, sodass keine deutliche Zunahme an Haushalten mit konsistenter Fernseherziehung festzustellen war. Für die Medienerziehung im Bereich der Video- und Computerspiele konnte jedoch eine deutliche Zunahme an Haushalten mit konsistenter Computerspielerziehung beobachtet werden. Vergleicht man diese beiden Trends getrennt nach Untersuchungsgruppen ergibt sich jedoch, insbesondere für das Fernsehen, ein etwas differenzierteres Bild. So ist vor allem in der Interventionsgruppe, in der zum ersten Halbjahr der dritten Klasse der geringste Anteil von Kindern mit konsistenter Fernseherziehung zu beobachten war, bis zum Ende der fünften Klasse eine deutliche Zunahme von Kindern, die über ein 
mittleres oder hohes regulatives Monitoring berichten, zu verzeichnen (vgl. Abbildung 88). Ende der sechsten Klasse fällt dieser Wert wieder leicht ab. In der Baselinegruppe bleibt dieser Anteil relativ stabil, in der Kontrollgruppe nimmt er sogar deutlich ab. Für die elterliche Erziehung im Bereich der Video- und Computerspiele kann bis zum Ende der fünften Klasse in allen Untersuchungsgruppen gleichermaßen eine deutliche Zunahme an Haushalten mit mittlerem oder hohem regulativen Monitoring beobachtet werden (vgl. Abbildung 89). Diese Zunahme ist aber mit 26 Prozent in der Interventionsgruppe ausgeprägter als in der Kontrollgruppe (21\%). Danach stagniert diese wiederum in allen Gruppen.

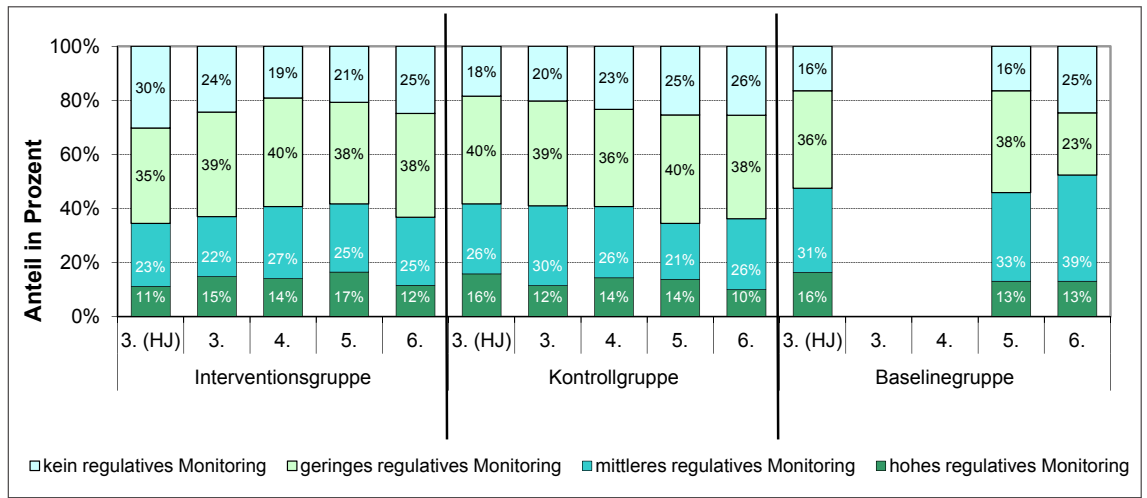

Abbildung 88. Elterliche Medienerziehung (Fernsehen) getrennt nach Jahrgangsstufe und Untersuchungsgruppe. ${ }^{241}$

$241 N=559$. Kontrollgruppe: k. A.t1 $=18, \mathrm{k} \cdot \mathrm{A}_{\cdot \mathrm{t} 2}=19, \mathrm{k} \cdot \mathrm{A}_{\cdot \mathrm{t} 3}=10, \mathrm{k} \cdot \mathrm{A}_{\cdot \mathrm{t} 4}=14, \mathrm{k} \cdot \mathrm{A}_{\cdot \mathrm{t} 5}=6$; Interventionsgruppe: $\mathrm{k} . \mathrm{A}_{\cdot \mathrm{t} 1}=17, \mathrm{k} \cdot \mathrm{A}_{\cdot \mathrm{t} 2}=6, \mathrm{k} \cdot \mathrm{A}_{\cdot \mathrm{t} 3}=8, \mathrm{k} \cdot \mathrm{A}_{\cdot \mathrm{t} 4}=7, \mathrm{k} \cdot \mathrm{A}_{\cdot \mathrm{t} 5}=17$; Baselinegruppe: $\mathrm{k} \cdot \mathrm{A}_{\cdot \mathrm{t} 1}=3, \mathrm{k} \cdot \mathrm{A}_{\cdot \mathrm{t} 4}=3, \mathrm{k} \cdot \mathrm{A}_{\cdot \mathrm{t} 5}=3$. 


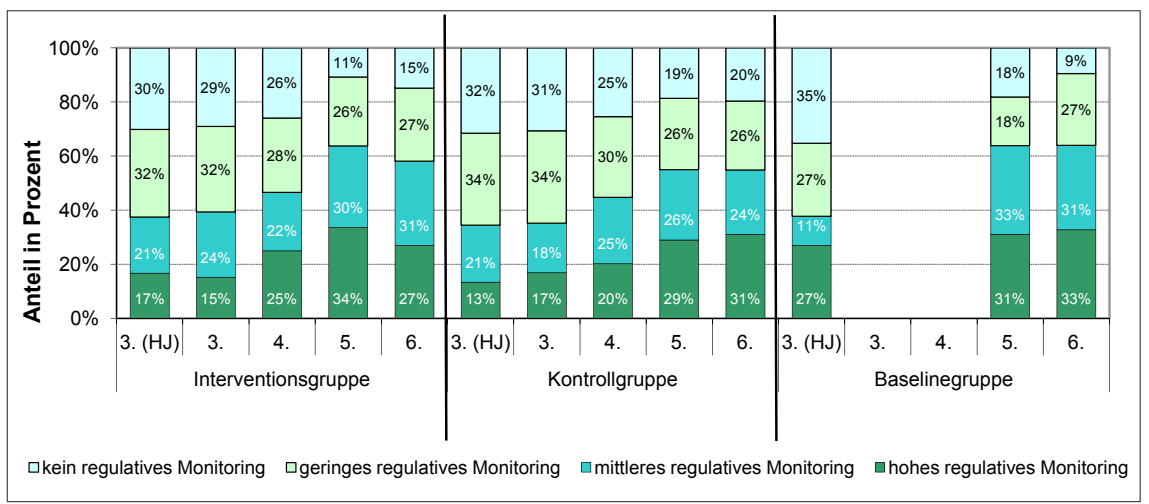

Abbildung 89. Elterliche Medienerziehung (Computerspiele) getrennt nach Jahrgangsstufe und Untersuchungsgruppe. ${ }^{242}$

Für die elterliche Interneterziehung sind insgesamt keine Unterschiede zwischen den Gruppen und Messzeitpunkten (5. und 6. Klasse) festzustellen (vgl. Abbildung 90). Eine Multivariate (TV, Computerspiele) Varianzanalyse mit Messwiederholung - 5 (Messzeitpunkt) x 2 (Gruppe) x 3 (Bildungsniveau) x 2 (Geschlecht) -, für die beiden Erziehungsbereiche, zu denen zu allen Messzeitpunkten Daten vorlagen, zeigte zudem, dass der beobachtete Treatmenteffekt zwar vom Geschlecht der Schülerinnen und Schüler unabhängig, aber vom Bildungsniveau im Elternhaus abhängig ist (Messzeitpunkt $F(8 / 279)=10.87, p<$. $01, f=.56$; Bildungsniveau $F(4 / 572)=3.21, p<.01, f=.15$; Gruppe $F(2 / 285)=1.46, p=.23$; Geschlecht $F(2 / 285)=.51, p=.60$; Bildungsniveau * Zeit $F(16 / 560)=1.73, p<.05, f=.12$; Bildungsniveau * Zeit * Gruppe $F(16 / 560)=2.37, p<.01, f=.25$; keine weiteren bedeutsamen Interaktionen).

$242 N=559$. Kontrollgruppe: k. A.t1 $=37, \mathrm{k} \cdot \mathrm{A}_{\cdot \mathrm{t} 2}=27, \mathrm{k} \cdot \mathrm{A}_{\cdot \mathrm{t} 3}=18, \mathrm{k} \cdot \mathrm{A}_{\cdot \mathrm{t} 4}=15, \mathrm{k} \cdot \mathrm{A}_{\cdot \mathrm{t} 5}=11$;

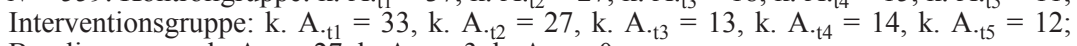
Baselinegruppe: $\mathrm{k} \cdot \mathrm{A}_{\cdot \mathrm{t} 1}=27, \mathrm{k} \cdot \mathrm{A}_{\cdot \mathrm{t} 4}=3, \mathrm{k} \cdot \mathrm{A}_{\cdot \mathrm{t5}}=0$. 


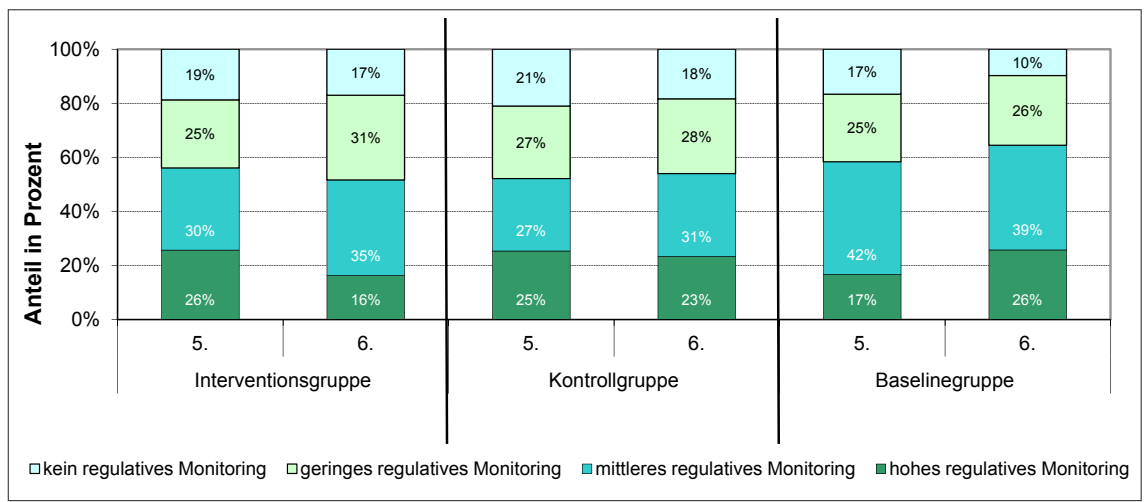

Abbildung 90. Elterliche Medienerziehung (Internet) getrennt nach Jahrgangsstufe und Untersuchungsgruppe. ${ }^{243}$

Nachgeschaltete univariate Varianzanalysen zeigten darüber hinaus, dass dieser Einfluss des Bildungshintergrundes gleichermaßen für regulatives Monitoring im Bereich des Fernsehens wie für regulatives Monitoring im Bereich der Computerspiele zu beobachten ist. So sind hinsichtlich der elterlichen Medienerziehung für Kinder aus Elternhäusern mit niedrigem Bildungsniveau (der höchste Bildungsabschluss beider Elternteile ist der Hauptschulabschluss) keine unterschiedlichen Verläufe in Abhängigkeit des durchgeführten medienpädagogischen Unterrichts zu beobachten (vgl. Abbildung 91, dargestellt ist das mittlere regulative Monitoring im Elternhaus).

$243 N=559$. Kontrollgruppe: k. A. $\cdot_{t 4}=22, \mathrm{k} \cdot \mathrm{A}_{\cdot t 5}=11$; Interventionsgruppe: $\mathrm{k} \cdot \mathrm{A}_{\cdot 44}=19$, $\mathrm{k}$. $\mathrm{A}_{\mathrm{t} 5}=3$; Baselinegruppe: $\mathrm{k}$. A.t $=4, \mathrm{k} \cdot \mathrm{A}_{\cdot \mathrm{t} 5}=2$. 

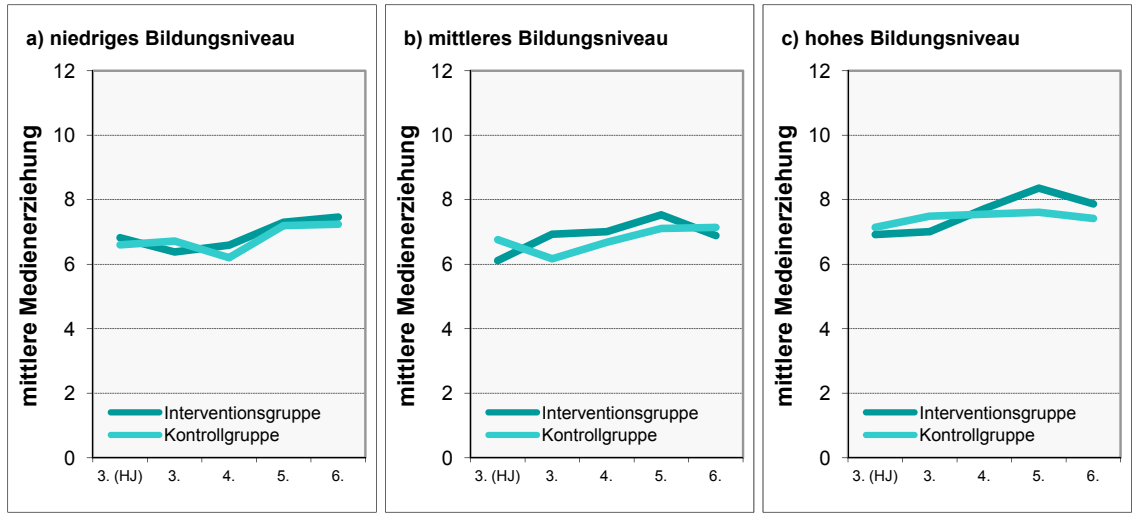

Abbildung 91. Elterliche Medienerziehung (Internet) getrennt nach Jahrgangsstufe Untersuchungsgruppe und Bildungsniveau.

Für Kinder aus Familien mit mittlerem Bildungsniveau (der höchste Bildungsabschluss mindestens eines Elternteils ist die Mittlere Reife) zeigen sich vor allem kurz- und mittelfristige Erfolge des Unterrichtsprogramms. Schülerinnen und Schüler der Interventionsgruppe aus Familien mit hohem Bildungsniveau (der höchste Bildungsabschluss mindestens eines Elternteils ist das Abitur oder ein abgeschlossenes Studium) berichten vor allem langfristig über verstärkte medienerzieherische Bemühungen in ihrem Elternhaus. Somit profitieren hinsichtlich eines regulativen Monitorings im Elternhaus insgesamt vor allem Kinder aus Elternhäusern mit mittlerem oder hohem Bildungsniveau von dem durchgeführten Medienunterrichtsprogramm (d. h. Elternabend).

Somit konnten bei Betrachtung der Entwicklung der kindlichen Mediennutzung hinsichtlich Bildschirmmedienausstattung im Kinderzimmer, Bildschirmmedienzeiten, konsumierter Bildschirmmedieninhalte sowie elterlicher Medienerziehung erste Erfolge des durchgeführten Medienunterrichtsprogrammes berichtet werden. Erfolge, die sich nicht nur im Mediennutzungsverhalten der Kinder, sondern auch zum Teil in einer Sensibilisierung des Umfeldes, im Rahmen der elterlichen Medienerziehung, manifestieren. Von entscheidendem Interesse ist nun die Frage, ob diese Unterschiede auch in weiteren Bereichen, wie zum Beispiel einer unterschiedlichen Entwicklung der Schulleistungen, ihren Niederschlag finden. 


\subsubsection{Schulleistungsentwicklung im Gruppenvergleich}

In den Gruppenvergleich wurden nur die Noten, gemessen am Ende der dritten, vierten, fünften und sechsten Klasse aufgenommen. Dies geschah aus Gründen der Vergleichbarkeit. Die Noten, die am Ende des jeweiligen Schuljahres erhoben wurden, sind immer die Halbjahresnoten, die zeitlich vor Beginn des Medienunterrichts liegen, da dieser jeweils nach den Halbjahreszeugnissen durchgeführt wurde. Zudem liegen zum ersten Messzeitpunkt nur zu 60 Prozent der Schülerinnen und Schüler Benotungen vor.

Insgesamt verschlechtern sich die mittleren Noten in allen erhobenen Fächern (mit Ausnahme von Sport) von der dritten bis zur sechsten Klasse (vgl. Kapitel 3.2.1), was unter anderem auf eine stärkere Leistungsdifferenzierung, speziell im unteren Leistungsbereich, zurückzuführen ist. Betrachtet man aber die Untersuchungsgruppen getrennt, so zeigt sich, dass Kontroll- und Interventionsklassen sich bis zum Ende der vierten Klasse zwar vergleichbar verschlechtern. Danach bremst sich dieser Trend jedoch in den Interventionsklassen deutlich ab, während er sich in den Kontrollklassen fortsetzt, d. h. in den Interventionsklassen verschlechtern sich die Noten zu einem geringeren Ausmaß (vgl. Abbildung 92, die Noten gemessen zum Halbjahr der dritten Klasse wurde hier als Vergleichswert für die Baselinegruppe mit aufgenommen, da in dieser erst wieder in der fünften Klasse gemessen wurde). Für eine genauere Analyse möglicher Effekte des durchgeführten Unterrichtsprogrammes auf die Schulleistungsentwicklung der Schülerinnen und Schüler wurde für die mittleren Noten in den Fächern Deutsch, Mathematik und Sachkunde (dritte und vierte Klasse) bzw. Englisch (fünfte und sechste Klasse) eine Varianzanalyse mit Messwiederholung - 4 (Messzeitpunkt) x 2 (Gruppe) x 2 (Geschlecht) und der Kovariaten IQ der Kinder (CFT 20 gemessen in der fünften Klasse) -, berechnet (Messzeitpunkt $F(3 / 1149)=4.71$, $p<.01, f=.11$; Gruppe $F(1 / 383)=7.24, p<.01, f=.14$; Geschlecht $F(1 / 383)=$. $89, p=.14$; CFT $F(1 / 383)=115.98, p<.01, f=.55$; Zeit $*$ Geschlecht $F(3 / 1149)=9.19, p<.01, f=.15$; Zeit * Geschlecht * Gruppe $F(3 / 1149)=3.57$, $p<.05, f=.09$; keine weiteren bedeutsamen Interaktionen). Die durchgeführte Analyse zeigte, dass der Treatmenteffekt zwar unabhängig von den kognitiven Fähigkeiten der Kinder ist, aber zusätzlich durch das Geschlecht moderiert wird. Eine ebenfalls beobachtete Interaktion zwischen Messzeitpunkt und Geschlecht zeigte sich bereits bei Beschreibung der Schulleistungsentwicklung in Kapitel 3.2.1 (vgl. Abbildung 50) und ist noch einmal für die mittleren Noten in Abbildung 93 dargestellt. Demnach öffnet sich die Schere in den Notenunterschieden zwischen Jungen und Mädchen mit der Zeit zunehmend zuungunsten der Jungen. Darüber hinaus zeigt Abbildung 93 ebenfalls recht deutlich, dass der beobachtete Treatmenteffekt vor allem auf die Mädchen zurückzuführen ist. So verschlech- 
tern sich Jungen beider Gruppen gleichermaßen, während dieser Trend für die Mädchen der Interventionsgruppe ab der vierten Klasse stagniert und für die Mädchen der Kontrollgruppe zwischen der vierten und fünften Klasse abgeschwächt wird.

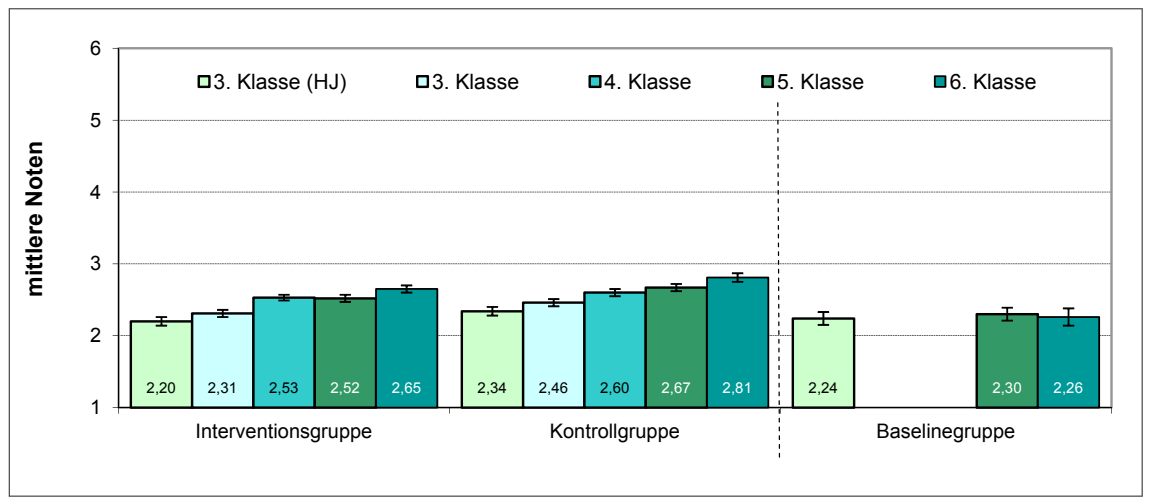

Abbildung 92. Mittlere Noten getrennt nach Jahrgangsstufe und Untersuchungsgruppe. ${ }^{244}$
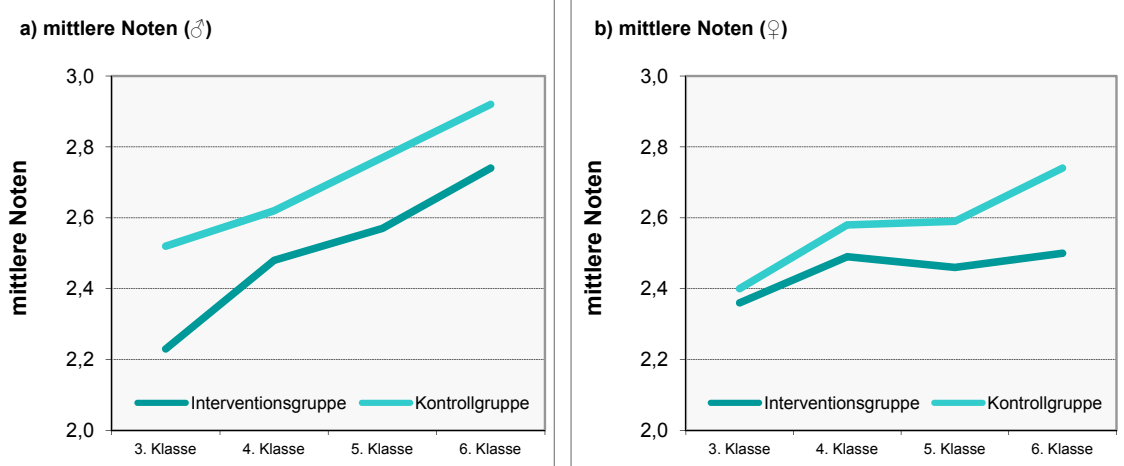

Abbildung 93. Mittlere Noten getrennt nach Jahrgangsstufe, Untersuchungsgruppe und Geschlecht.

$244 N=559$. Kontrollgruppe: $\mathrm{k} \cdot \mathrm{A}_{\cdot \mathrm{t} 1}=103, \mathrm{k} \cdot \mathrm{A}_{\cdot \mathrm{t} 2}=30, \mathrm{k} \cdot \mathrm{A}_{\cdot \mathrm{t} 3}=42, \mathrm{k} \cdot \mathrm{A}_{\mathrm{t}_{4}}=29$, $\mathrm{k} . \mathrm{A}_{\cdot t 5}=39$; Interventionsgruppe: $\mathrm{k} \cdot \mathrm{A}_{\cdot \mathrm{t} 1}=99, \mathrm{k} \cdot \mathrm{A}_{\cdot \mathrm{t} 2}=14, \mathrm{k} \cdot \mathrm{A}_{\cdot \mathrm{t} 3}=1, \mathrm{k} \cdot \mathrm{A}_{\cdot \mathrm{t} 4}=10$, $\mathrm{k} . \mathrm{A}_{\cdot \mathrm{t} 5}=2$; Baselinegruppe: $\mathrm{k} . \mathrm{A}_{\mathrm{t} 1}=19, \mathrm{k} \cdot \mathrm{A}_{\cdot \mathrm{t} 4}=11, \mathrm{k} \cdot \mathrm{A}_{\cdot \mathrm{t} 5}=22$. 


\subsubsection{Entwicklung des Gewaltverhaltens im Gruppenvergleich}

Zur Beurteilung der Entwicklung des Gewaltverhaltens in den unterschiedlichen Untersuchungsgruppen soll dieses analog zu Kapitel 4.2.1 getrennt nach psychischer Gewalt und Mobbing in der Schule sowie delinquentem Verhalten außerhalb der Schule betrachtet werden. Es ist zu beachten, dass das Gewaltverhalten der Schülerinnen und Schüler erstmalig in der vierten Klasse zu t 3 erhoben wurde, und somit bereits zwei Einheiten des Medienunterrichts durchgeführt wurden. Etwaige Unterschiede zwischen den Untersuchungsgruppen können aber nur dann auf das durchgeführte Medienunterrichtsprogramm zurückgeführt werden, wenn sich die beiden Untersuchungsgruppen zu t 1 nicht unterschieden haben. Neben vergleichbaren Gewaltprävalenzwerten in der vierten Klasse, die ähnliches für die dritte Klasse vermuten lassen, unterscheiden sich die beiden Untersuchungsgruppen statistisch nicht bedeutsam hinsichtlich der Einflussvariablen (gemessen zu t 3) Empathie und Risikosuche, den Medienvariablen (gemessen zu t 1) Ausstattung, Nutzungszeiten, Gewaltmediennutzung (vgl. Kapitel 7.2.2) sowie den soziodemographischen Variablen Geschlecht, Bildungsniveau und Migrationshintergrund. Geht man von einer eigenständigen Wirkung des durchgeführten Medienunterrichtsprogrammes auf die Variablen der Mediennutzung (vgl. Kapitel 7.2.2) sowie einem Einfluss der Mediennutzung auf das Gewaltverhalten (vgl. Kapitel 4.2.2, 4.2.3) aus, können Gruppenunterschiede im beobachteten Gewaltverhalten zumindest auch zum Teil auf das Medienunterrichtsprogramm zurückgeführt werden.

Schulgewalt. Hinsichtlich Gewalt in der Schule haben zum Ende der vierten Klasse 16 Prozent aller Kinder angegeben, innerhalb der letzten vier Wochen Täter von Schulgewalt ${ }^{245}$ gewesen zu sein (Interventionsgruppe: $14 \%$, Kontrollgruppe: $16 \%$ ). Bis zum Ende der sechsten Klasse erhöht sich die Prävalenz von Schulgewalt insgesamt auf 20 Prozent (Interventionsgruppe: 18\%, Kontrollgruppe: $22 \%$ ), wobei die Zunahme in der Kontrollgruppe um zwei Prozentpunkte höher ausfällt. In beiden Untersuchungsgruppen ist jedoch in der fünften Klasse ein deutlicher (Interventionsgruppe) bzw. leichter (Kontrollgruppe) Rückgang der Prävalenzzahlen festzustellen (vgl. Abbildung 94, siehe auch Abbildung 61). Diese deskriptiven Gruppenunterschiede werden allerdings nur zum Teil statistisch bedeutsam. Zunächst zeigt sich beim direkten Vergleich von Kontroll- und Interventionsklassen ${ }^{246}$ in beiden Gruppen eine statistisch bedeutsame Verände-

245 Dargestellt ist der Anteil der Schülerinnen und Schüler, welcher innerhalb der letzten 4 Wochen mindestens einmal (,1- oder 2-mal“, „,3- bis 6-mal“, ,noch häufiger“) Täter von Gewalt in der Schule gewesen ist.

246 Die Baseline-Klassen wurden aufgrund der deutlich geringeren Stichprobengröße und dem Vorliegen von Daten zu lediglich zwei Messzeitpunkten aus den Signifikanzprüfungen ausgeschlossen. 
rung der Schulgewalt über die Zeit (Interventionsgruppe: Cochrans- $Q(2, N=$ $236)=10.96, p<.01$; Kontrollgruppe: Cochrans $Q(2, N=227)=6.64, p<.05)$. Die Schülerinnen und Schüler der beiden Gruppen unterscheiden sich jedoch bei keinen bestehenden Gruppenunterschieden zu t $3\left(\chi^{2}(1, \mathrm{~N}=478)=.40, p=\right.$. $53)$ - lediglich zu Messzeitpunkt t $4(\chi 2(1, N=483)=4.56, p<.03)$ und nicht zu t $5\left(\chi^{2}(1, \mathrm{~N}=492)=1.13, p=.29\right)$ statistisch bedeutsam voneinander.

Schulmobbing. Formen des Mobbings ${ }^{247}$ in der Schule kommen insgesamt deutlich häufiger vor. Zum Ende der vierten Klasse haben bereits 41 Prozent der Kinder angegeben innerhalb der letzten vier Wochen Täter von Schulmobbing gewesen zu sein (Interventionsgruppe: $38 \%$, Kontrollgruppe: $42 \%$ ). Bis zum Ende der sechsten Klasse erhöht sich die Prävalenz von Mobbing in der Schule insgesamt auf 48 Prozent (Interventionsgruppe: 49\%, Kontrollgruppe: 49\%, Baselinegruppe: $39 \%$ ) wobei die deutlichste Zunahme in den Angaben zum Hänseln festzustellen ist (vgl. Abbildung 94, siehe auch Abbildung 61). Gruppenunterschiede sind lediglich in einem Rückgang der Prävalenzzahlen in der Kontrollgruppe in der fünften Klasse festzustellen. ${ }^{248}$

Gewaltdelinquenz außerhalb der Schule. Als Gewaltdelinquenz wurde gewertet, wenn ein Kind in einem der beiden Bereiche Sachbeschädigung oder Verletzen/Drohen innerhalb der letzten 12 Monate auffällig war. Zum Ende der vierten Klasse waren dies 21 Prozent der Kinder (Interventionsgruppe: 19\%, Kontrollgruppe: $22 \%$ ). Vergleicht man die Angaben, die Ende der vierten Klasse gemacht wurden, mit den Angaben vom Ende der sechsten Klasse (vgl. Abbildung 94, siehe auch Abbildung 62), fällt einem zunächst ein Rückgang der Gewalttäter außerhalb der Schule auf insgesamt 18 Prozent auf (Interventionsgruppe: $19 \%$, Kontrollgruppe: $17 \%$, Baselinegruppe: 17\%). Dieser findet allerdings lediglich in der Kontrollgruppe seine Ursache. ${ }^{249}$ Gruppenunterschiede sind aber auch in einem Rückgang der Prävalenzzahlen in der Interventionsgruppe bei einer gleichzeitigen Zunahme in der Kontrollgruppe in der fünften Klasse festzustellen. ${ }^{250}$

247 Dargestellt ist der Anteil der Schülerinnen und Schüler, welcher innerhalb der letzten 4 Wochen mindestens einmal (,1- oder 2-mal“, ,,3- bis 6-mal“, „,noch häufiger“) Täter von Mobbing in der Schule gewesen ist.

248 Interventionsgruppe: Cochrans- $Q(2, \mathrm{~N}=236)=8.79, p<.01$; Kontrollgruppe: Cochrans $Q(2, \mathrm{~N}=227)=3.30, p=.19 . \mathrm{t} 3: \chi^{2}(1, \mathrm{~N}=478)=.77, p=.38 ; \mathrm{t} 4: \chi^{2}(1, \mathrm{~N}=483)$ $=1.66, p=.20 ; \mathrm{t} 5: \chi^{2}(1, \mathrm{~N}=492)=.01, p=.93$.

249 Interventionsgruppe: Cochrans- $Q(2, \mathrm{~N}=237)=3.44, p=.18$; Kontrollgruppe: Cochrans $Q(2, \mathrm{~N}=223)=7.20, p<.05$.

$250 \mathrm{t} 3: \chi^{2}(1, \mathrm{~N}=478)=.50, p=.48 ; \mathrm{t} 4: \chi^{2}(1, \mathrm{~N}=482)=11.25, p<.01 ; \mathrm{t} 5: \chi^{2}(1, \mathrm{~N}=490)$ $=.49, p=.48$. 


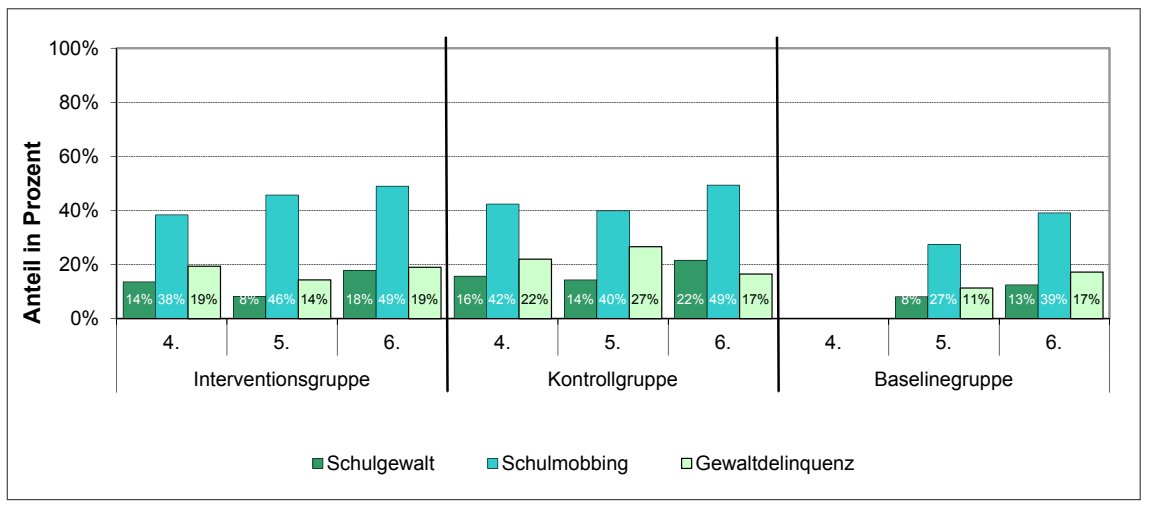

Abbildung 94. Täterraten selbstberichteter Delinquenz innerhalb und außerhalb der Schule nach Jahrgangsstufe und Untersuchungsgruppe.

Zusammengefasst finden sich die deutlichsten Gruppenunterschiede in einem abweichenden Verlauf der Entwicklung von Schulgewalt und Gewaltdelinquenz außerhalb der Schule in Kontroll- und Interventionsgruppe. Für beide Formen der Gewalt fallen die Prävalenzzahlen in der fünften Klasse in der Interventionsgruppe deutlich geringer aus, wobei sie in der Kontrollgruppe ansteigen (Gewaltdelinquenz) bzw. nur leicht zurückgehen (Schulgewalt).

Eine nachfolgende getrennte Überprüfung auf Treatmentsensitivität erbrachte keine bedeutsamen Unterschiede zwischen Bildungsniveau im Elternhaus bzw. Geschlecht der Kinder und einer Untersuchungsgruppenzugehörigkeit.

\subsubsection{Problematisches Computerspielverhalten im Gruppenvergleich}

Problematisches Computerspielverhalten wurde mittels der KFN-CSAS in der fünften und sechsten Klasse erhoben. ${ }^{251}$ Neben einer Einteilung nach computer-

251 Da Problematisches Computerspielverhalten der Schülerinnen und Schüler erstmalig erst nach den Medienunterrichtseinheiten erhoben wurde, gilt auch hier wie für das Gewaltverhalten, dass etwaige Unterschiede zwischen den Untersuchungsgruppen nur dann auf das durchgeführte Medienunterrichtsprogramm zurückgeführt werden können, wenn sich die beiden Untersuchungsgruppen zu t 1 nicht unterschieden haben. Die beiden Untersuchungsgruppen unterscheiden sich zunächst statistisch nicht bedeutsam hinsichtlich der Medienvariablen (gemessen zu t 1) Nutzungszeiten und Gewaltmediennutzung (vgl. Kapitel 7.2.2) sowie den soziodemographischen Variablen Geschlecht, Bildungsniveau und Migrationshintergrund. Geht man von einem deutlichen Zusammenhang der Nutzungszeiten ( $\mathrm{t} 1$ ) sowie der Gewaltmediennutzung ( $\mathrm{t} 1$ ) mit späterem problematischen Computerspielverhalten (vgl. Kapitel 5.2.3, 5.2.4), sowie einer eigenständigen Wirkung des durchgeführten Medienunterrichtsprogrammes auf die Variablen der Mediennutzung 
spielabhängigen bzw. gefährdeten Spielern, soll auch die Summenvariable zur Computerspielabhängigkeit ${ }^{252}$ im Gruppenvergleich betrachtet werden (vgl. Kapitel 5.2.4). Diese kann auf einem Kontinuum von unproblematischem hin zu problematischem Computerspielverhalten als Maß für die Tendenz, dass eine befragte Person für abhängiges Computerspielen anfällig ist, betrachtet werden.

Insgesamt steigt der Anteil der Kinder mit problematischen Computerspielverhalten von der fünften zur sechsten Klasse (vgl. Kapitel 5.2.2), wobei sich die beiden Untersuchungsgruppen bereits in der fünften Klasse deutlich unterscheiden (vgl. Abbildung 96). Für eine genauere Analyse möglicher Effekte des durchgeführten Unterrichtsprogrammes auf die Entwicklung problematischen Computerspielverhaltens der Schülerinnen und Schüler wurde für die Summenvariable problematischer Computerspielnutzung eine Varianzanalyse mit Messwiederholung - 2 (Messzeitpunkt) x 2 (Gruppe) x 2 (Geschlecht) -, berechnet. Die deskriptiv beobachteten Unterschiede manifestieren sich dabei in zwei statistisch bedeutsamen Haupteffekten Gruppe $(F(1 / 407)=3.89, p<.05, f=.10)$ und Messzeitpunkt $(F(1 / 407)=6.41, p<.05, f=.13)$. Zusätzlich zeigt sich noch einmal eine Abhängigkeit problematischen Computerspielverhaltens vom Geschlecht der Kinder $F(1 / 407)=53.74, p<.01, f=.36)$, die bereits in Kapitel 5.2 dokumentiert werden konnte. ${ }^{253}$

(vgl. Kapitel 7.2.2) aus, können Gruppenunterschiede zumindest auch zum Teil auf das Medienunterrichtsprogramm zurückgeführt werden.

252 Aus Gründen der Vergleichbarkeit der Daten der fünften und sechsten Klasse gingen allerdings in die Summenbildung wiederum nur die zehn Variablen ein, die zu beiden Messzeitpunkten abgefragt wurden (vgl. Kapitel 5.2.5).

253 Es konnten keine statistisch bedeutsamen Interaktionen beobachtet werden. Somit können Veränderung zwischen t 4 und t 5 nicht auf etwaige Unterrichtseffekte zurückgeführt werden. 

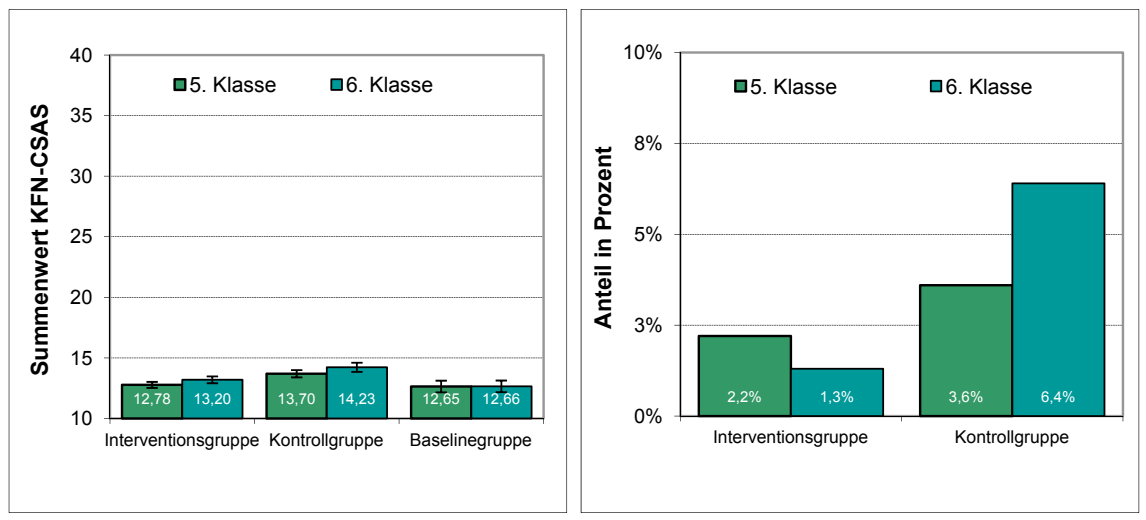

Abbildung 95. Problematisches Computerspielverhalten und

Computerspielabhängigkeit (Anteil gefährdet bzw. abhängig) nach Erhebungszeitpunkt und Untersuchungsgruppe. In der Baselinegruppe liegt der Anteil der Gefährdeten bzw. Abhängigen in der fünften und sechsten Klasse bei 0 Prozent.

Ähnliches gilt, wenn man den - gemäß der KFN-CSAS eingeteilten - Anteil computerspielabhängiger und gefährdeter Schülerinnen und Schüler in den Untersuchungsgruppen betrachtet (vgl. Abbildung 96): So unterscheiden sich die beiden Untersuchungsgruppen wiederum bereits in der fünften Klasse. ${ }^{254}$ Danach vergrößert sich der Anteil jedoch in der Kontrollgruppe, ${ }^{255}$ wohingegen er in der Interventionsgruppe ${ }^{256}$ abnimmt. ${ }^{257}$ Somit zeigt sich bei Betrachtung des Anteils gefährdeter bzw. computerspielabhängiger Schülerinnen und Schüler auch ein längerfristiger Effekt des durchgeführten Medienunterrichtsprogrammes. ${ }^{258}$

\subsubsection{Entwicklung von Fettleibigkeit im Gruppenvergleich}

Insgesamt ist von der dritten bis zur sechsten Klasse eine Zunahme derjenigen Schülerinnen und Schüler, die als übergewichtig oder fettleibig eingestuft werden müssen, zu beobachten. Diese Zunahme scheint in der Kontrollgruppe etwas

254 Dieser Unterschied wird allerdings statistisch nicht bedeutsam: $\chi^{2}(1, \mathrm{~N}=450)=.74$, $p=39$.

255 Cochrans $-Q(1, \mathrm{~N}=205)=5.44, p<.05$.

256 Cochrans $-Q(1, \mathrm{~N}=206)=.67, p=.41$.

$257 \chi^{2}(1, \mathrm{~N}=443)=7.66, p<.01$.

258 Eine Vergleichbarkeit der Untersuchungsgruppen zu t 1 muss für diese Schlussfolgerung allerdings vorausgesetzt werden. Eine Untersuchung auf systematische Treatmentsensitivität kann hier aufgrund der geringen Fallzahlen nicht durchgeführt werden. 
höher auszufallen (vgl. Abbildung 96), so zeigt sich in dieser, bei vergleichbaren Ausgangwerten mit den Interventionsklassen ( $1: \chi^{2}(1, N=222)=.18, p=.67$ ), ein statistisch bedeutsamer stetiger Zuwachs an übergewichtigen oder fettleibigen Kindern (t 1 bis t 5: Cochrans- $Q(4, N=208)=14.50, p<.01)$. In der Interventionsgruppe stagnieren im Vergleich dazu die Zahlen annähernd (t 1 bis $\mathrm{t} 5$ : Cochrans- $Q(4, N=222)=85.48, p<.31)$. Die beiden Untersuchungsgruppen unterscheiden sich allerdings zu keinem der fünf Messzeitpunkte statistisch bedeutsam voneinander ( $\mathrm{t} 2: \chi^{2}(1, N=473)=.52, p=.47 ; \mathrm{t} 3: \chi^{2}(1, N=483)=$ 1.37, $p=.24 ; \mathrm{t} 4: \chi^{2}(1, N=474)=2.90, p=.09$; t 5: $\chi^{2}(1, N=493)=.92, p=$. $34)$.

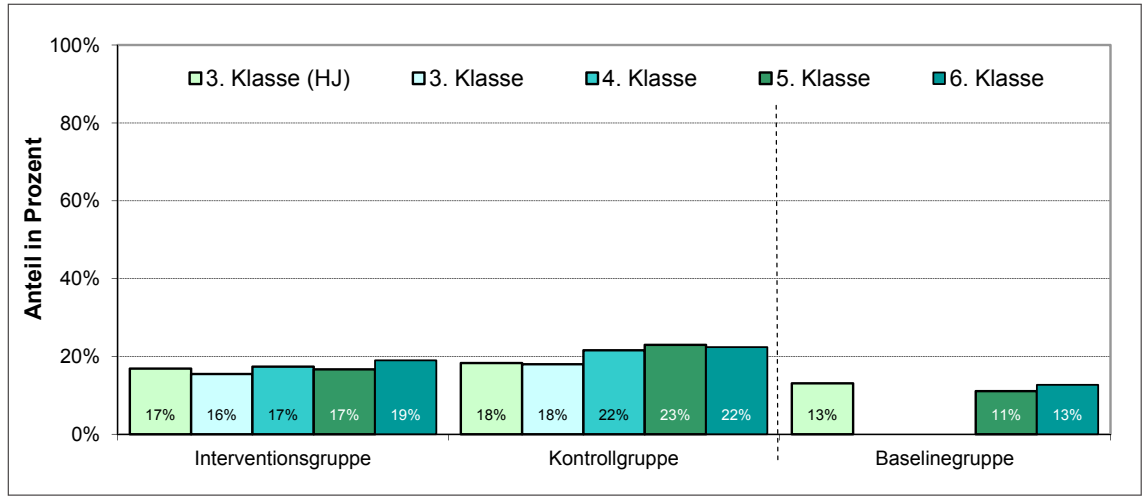

Abbildung 96. Anteil übergewichtiger und adipöser Kinder nach Jahrgangsstufe und Untersuchungsgruppe.
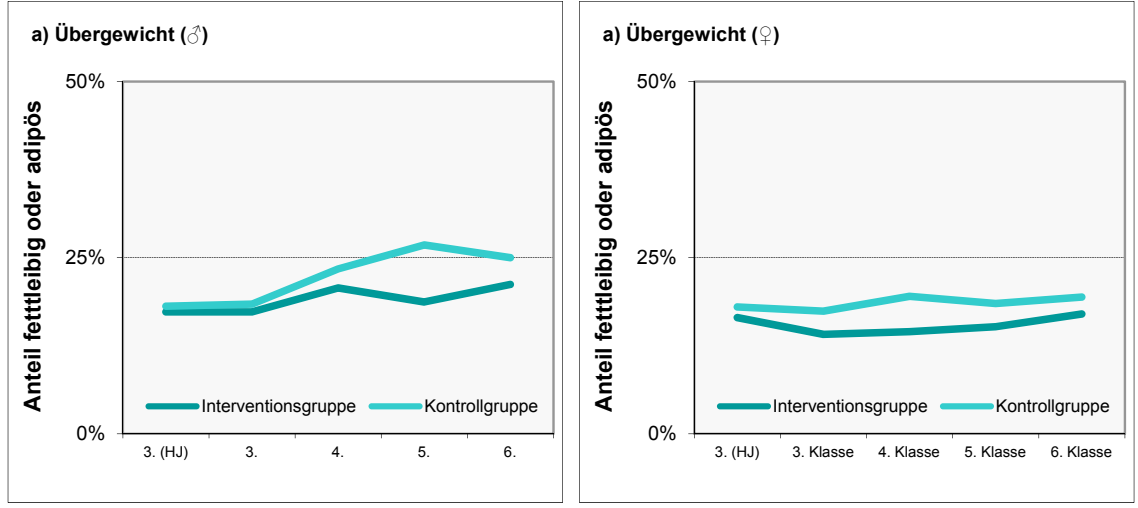

Abbildung 97. Anteil übergewichtiger und adipöser Kinder nach Geschlecht, Jahrgangsstufe und Untersuchungsgruppe. 
Für eine nachgeschaltete Überprüfung einer geschlechtsspezifischen Treatmentsensitivität wurden obige Analysen getrennt für Jungen und Mädchen berechnet (vgl. Abbildung 97). Unter den Jungen nahm der Anteil derer, die als übergewichtig oder fettleibig klassifiziert werden müssen, lediglich in der Kontrollgruppe von der dritten bis zur sechsten Klasse bedeutsam zu (Intervention: Cochrans- $Q(4, N=99)=4.24, p=.37$; Kontrolle: Cochrans $Q(4, N=$ $114)=15.84, p<.01)$. Für die Mädchen ist in keiner der Gruppen eine statistisch bedeutsame Zunahme zu beobachten (Intervention: Cochrans- $Q \quad(4, N=$ $123)=3.18, p=.53$; Kontrolle: Cochrans- $Q(4, N=94)=3.06, p=.55)$. Die beiden Untersuchungsgruppen unterscheiden sich allerdings auch bei einer nach Geschlecht getrennten Betrachtung zu keinem der fünf Messzeitpunkte statistisch bedeutsam voneinander, weder für die Mädchen (t 1: $\chi^{2}(1, N=244)=.09, p=$. 76; t 2: $\chi^{2}(1, N=238)=.52, p=.47$; t $3: \chi^{2}(1, N=244)=1.07, p=.30 ; \mathrm{t} 4: \chi^{2}$ $\left.(1, N=240)=.49, p=.49 ; \mathrm{t} 5: \chi^{2}(1, N=248)=.25, p=.62\right)$ noch für die Jungen $\left(\mathrm{t} 1: \chi^{2}(1, N=239)=.07, p=.80 ; \mathrm{t} 2: \chi^{2}(1, N=235)=.05, p=.82 ; \mathrm{t} 3: \chi^{2}(1, N\right.$ $=239)=.25, p=.61 ; \mathrm{t} 4: \chi^{2}(1, N=234)=2.14, p=.14 ; \mathrm{t} 5: \chi^{2}(1, N=245)=.48$, $p=.49)$.

\subsection{Zusammenfassung der Evaluationsergebnisse und Diskussion}

Insgesamt konnte eine erfolgreiche Umsetzung, vor allem der ersten beiden medienpädagogischen Unterrichtseinheiten, des Medienpräventions- und Interventionsprogramms des Berliner Längsschnitts Medien erreicht werden. Das gewählte Curriculum und dessen didaktische Aufbereitung fand dabei bei Lehrkräften sowie Schülerinnen und Schülern zufällig ausgewählter Grundschulklassen gute Resonanz, wobei der Unterricht nicht in allen Klassen und auch nicht immer im vollen Umfang umgesetzt wurde. Das entwickelte Lehrmaterial lieferte aber zumindest einen für das Alter der Schülerinnen und Schüler geeigneten Materialpool, aus dem die Lehrkräfte je nach den gegebenen Rahmenbedingungen Unterrichtselemente auswählen konnten. Das Medienunterrichtsprogramm konnte dabei gut in den normalen Unterricht integriert werden, wobei dies meist in den beiden Fächern Deutsch und Sachkunde geschah.

Effekte des Unterrichtsprogramms zeigten sich nicht nur auf den primär fokussierten Variablen der Mediennutzung, sondern auch zum Teil auf den durch Mediennutzung beeinflussten Variablen Schulleistung, Gewaltverhalten, Computerspielabhängigkeit sowie Fettleibigkeit; Ergebnisse, die wie folgt zusammengefasst werden können:

- Hinsichtlich der Ausstattung mit Mediengeräten (Fernseher, Spielkonsole, PC) im Kinderzimmer konnten schwache mittelfristige und v. a. langfristige 
Effekte des durchgeführten Medienunterrichts erreicht werden: im Vergleich zur Kontrollgruppe konnten die Ausstattungszahlen in der Interventionsgruppe reduziert werden und zwar unabhängig vom Geschlecht der Kinder und dem Bildungsniveau im Elternhaus.

- Bezüglich der Mediennutzungszeiten konnten nur auf deskriptiver Ebene Erfolge des durchgeführten Medienunterrichtsprogrammes dahingehend erzielt werden, dass die Medienzeiten der Schülerinnen und Schüler der Interventionsgruppe im Vergleich zu den Medienzeiten der Schülerinnen und Schüler der Kontrollgruppe vorübergehend stagnierten. Diese Stagnation erreichte allerdings keine statistische Bedeutsamkeit.

- Zudem konnte das Medienunterrichtsprogramm mittelfristig (schwacher Effekt) die Nutzung problematischer Bildschirmmedieninhalte der Interventionsgruppe im Vergleich zur Kontrollgruppe reduzieren. Langfristig zeigen sich diese Effekt wiederum nur auf deskriptiver Ebene. Zudem reagierten Jungen dabei deutlich besser auf den durchgeführten Unterricht als Mädchen, v. a. hinsichtlich der Nutzung problematischer Fernsehinhalte.

- Die elterliche Medienerziehung betreffend konnten ebenfalls mittel- und langfristige Effekte des durchgeführten Medienunterrichtsprogramms erzielt werden, wobei vor allem Kinder aus Elternhäusern mit mittlerem oder hohem Bildungsniveau davon profitierten: Für Kinder aus Familien mit mittlerem Bildungsniveau zeigten sich vor allem kurz- und mittelfristige Erfolge des Unterrichtsprogramms. Schülerinnen und Schüler der Interventionsgruppe aus Familien mit hohem Bildungsniveau berichten vor allem langfristig über verstärkte medienerzieherische Bemühungen in ihrem Elternhaus.

- Hinsichtlich der Schulleistungsentwicklung entfaltete das Medienunterrichtsprogramm eine schwache Wirkung: Bei einer allgemeinen Verschlechterung der Noten über die Jahre konnte dieser Trend in der Interventionsgruppe leicht abgebremst werden, wobei vor allem Mädchen der Interventionsgruppe sich im Vergleich zu ihrer Gegengruppe weniger verschlechterten. Der Treatmenteffekt war unabhängig von den kognitiven Fähigkeiten der Schülerinnen und Schüler.

- Betrachtet man die Entwicklung des Gewaltverhaltens im Gruppenvergleich, kann - eine Vergleichbarkeit der Untersuchungsgruppen trotz fehlender Baselinemessung vorausgesetzt - allenfalls eine mittelfristige Reduktion des Gewaltverhaltens innerhalb und außerhalb der Schule (nicht jedoch für Mobbing) in der Interventionsgruppe zum Teil auf das durchgeführte Unterrichtsprogramm zurückgeführt werden.

- Das durchgeführte Medienunterrichtsprogramm hatte zudem - eine Vergleichbarkeit der Untersuchungsgruppen trotz fehlender Baselinemessung vorausgesetzt - einen schwachen langfristigen Effekt auf die Entstehung prob- 
lematischen Computerspielverhaltens: In der Interventionsgruppe lag der Anteil der computerspielabhängigen und gefährdeten Schülerinnen und Schüler bereits in der fünften Klasse unter dem der Kontrollgruppe. Dieser Unterschied vergrößerte sich zudem bis zur sechsten Klasse.

- Bezüglich der körperlichen Entwicklung konnte wiederum auf deskriptiver Ebene ein Einfluss des Medienunterrichtsprogrammes dahingehend festgestellt werden, dass der Anteil der fettleibigen und übergewichtigen Kinder in der Interventionsgruppe stagniert, wobei dieser in der Kontrollgruppe zulegt. Tendenziell profitieren vor allem Jungen von diesem Effekt.

Insgesamt konnten somit mit einem schulbasierten Medieninterventions- und Medienpräventionsprogramm, welches explizit und ausschließlich das kindliche Mediennutzungsverhalten thematisierte vor allem mittel- und langfristige Effekte hinsichtlich der Ausstattung mit Mediengeräten erzielt werden (vgl. Cantor \& Wilson, 2003; Kunczik \& Zipfel, 2004; Robinson, 1999; Kline, 2005; Epstein et al., 2008). Der Einfluss des Unterrichtsprogramms manifestierte sich darüber hinaus auf deskriptiver Ebene auch mittelfristig in geringeren Nutzungszeiten sowie mittel und langfristig in einer geringeren Nutzung problematischer Bildschirmmedieninhalte. Im Gegensatz zu Robinson (1999; vgl. Robinson et al., 2001) konnten mit dem durchgeführten Unterrichtsprogramm die Mediennutzungszeiten jedoch nur in geringem Maße beeinflusst werden. Dies mag wohl vor allem darin begründet sein, dass das SMART-Konzept von Robinson und Kollegen (1999; vgl. Robinson et al., 2001; siehe auch Kleimann, 2011) einem Verhaltenstraining entspricht, welchem eine besondere Effektivität hinsichtlich der zu beeinflussenden Variablen zugesprochen wurde (Cantor \& Wilson, 2003; vgl. Kunczik \& Zipfel, 2004). Das Medieninterventions- und Medienpräventionsprogramm des Berliner Längsschnitts Medien gründet dagegen vor allem auf reflexiven Elementen sowie einem kritischen Dialog zwischen Lehrkräften und Schülerinnen und Schülern (vgl. Kleimann, 2011). Hierdurch wurden vor allem längerfristige Wirkungen erhofft (vgl. Kunczik \& Zipfel, 2004; Rosenkoetter et al., 2004; siehe auch Mößle et al., 2010; Kleimann, 2011). Nur durch eine weitere Begleitung der Stichprobe wäre jedoch die Überprüfung möglich, welche langfristigen Veränderungen sich durch eine gezielte frühe medienpädagogische Intervention und zeitliche Reduktion der Mediennutzung erreichen lassen.

Vor allem konnte jedoch im Medienunterrichtsprogramm des Berliner Längsschnitts Medien die Eingangs formulierte Hoffnung erfüllt werden, mit schulbasierten Programmen Gruppen zu erreichen, die sonst nicht von Präventionsprogrammen profitieren können. So zeigten sich die Effekte auf die Mediennutzung unabhängig vom Bildungsniveau im Elternhaus. Dies trifft allerdings nicht auf das elterliche Medienerziehungsverhalten zu, da hier vor allem Kinder aus Familien mit mittlerem und hohem Bildungsniveau über verstärkte Bemühungen ihrer 
Eltern berichteten. Dieser Effekt mag vielleicht auch durch die breite öffentliche Diskussion zur Wirkung von Computerspielen auf Gewalt und Schulleistung und einer damit verbunden Sensibilisierung in der Bevölkerung - insbesondere der Hochgebildeten - verstärkt worden sein (vgl. Kapitel 2.3). Leider kann dabei die genaue Rolle des im Rahmen dieses Interventions- und Präventionsprogrammes durchgeführten Elternabends, insbesondere im Hinblick auf kurz- und mittelfristige Effekte im Mediennutzungsverhalten, nicht genau aufgeschlüsselt werden. So könnte man annehmen, dass diese Effekte insbesondere auf den durchgeführten Elternabend und den damit verbundenen Änderungen im elterlichen Medienerziehungsverhalten und weniger auf das medienpädagogische Unterrichtsprogramm zurückzuführen sind.

Wie aus der Befundlage solcher Studien zum Gewalt- und Gesundheitsverhalten erhofft, die schulbasierten Maßnahmen, welche allein eine Reduktion des täglichen Medienkonsums zum Ziel hatten, ohne dabei Gewalt oder Gesundheitsverhalten explizit zu thematisieren, eine Beeinflussung auch des Gewaltverhaltens oder des Gesundheitsverhaltens der Schülerinnen und Schüler attestiert (vgl. Robinson, 1999; Robinson et al., 2001; Epstein et al., 2008), konnten auch zum Teil auf den durch Mediennutzung beeinflussten Variablen Schulleistung, Gewaltverhalten, Computerspielabhängigkeit sowie Fettleibigkeit Erfolge durch das durchgeführte Unterrichtsprogramm erzielt werden. Diese Effekte konnten im Berliner Längsschnitt Medien erstmalig auch für Schulleistungen berichtet werden. Vor allem die Mädchen der Interventionsgruppe verschlechterten sich hier am wenigsten. Da sich Mädchen und Jungen allerdings hinsichtlich der Medienparameter in der Wirkung des medienpädagogischen Unterrichtsprogrammes statistisch nicht bedeutsam unterschieden, stellt sich hier die Frage, ob eine besonders gefährdete Risikopopulation - Jungen -, mit schon zu Beginn „höheren“ Werten auf allen Medienvariablen, schlechter durch das Unterrichtsprogramm erreicht wurde oder ob hier andere Hintergrundfaktoren, welche durch den medienpädagogischen Unterricht beeinflusst wurden, eine Rolle spielen.

Zugegebener Maßen sind einige dieser Effekte nur schwach ausgeprägt und vor allem mittelfristig (Gewaltverhalten und Schulleistung) zu beobachten. Da das Gewaltverhalten der Schülerinnen und Schüler erst ab der vierten Klasse sowie das Thema der Computerspielabhängigkeit erst ab der fünften Klasse in der Befragung berücksichtigt wurde, kann eine weitere längsschnittliche Untersuchung risikobehafteter Nutzungsgewohnheiten und -präferenzen in ihren Auswirkungen auf akademische Performanz, Gewaltverhalten, Computerspielabhängigkeit sowie Fettleibigkeit von Kindern und Jugendlichen hier besonders gewinnbringend sein. Dies ist vor allem vor dem Hintergrund einer größeren Leistungsvarianz im Sekundarschulbereich sowie einer erhöhten Auftretenswahr- 
scheinlichkeit von Gewaltdelinquenz und Computerspielabhängigkeit im Jugendalter zu erwarten.

Zusammengefasst entwickelte das schulbasierte Medieninterventions- und Medienpräventionsprogramm des Berliner Längsschnitts Medien Wirkungen und dies obwohl es in einigen Klassen nicht optimal implementiert wurde und obwohl die Eltern als wichtige Multiplikatoren zum Teil nur schlecht erreicht wurden. Eine Schwierigkeit weiterer Bemühungen besteht allerdings noch immer darin, auch jene Gruppen zu erreichen, welche insbesondere von Präventionsprogrammen profitieren könnten, so zum Beispiel Kinder aus Familien mit niedrigem Bildungsniveau und geringer elterlicher Zuwendung. Da die Teilnahme an Gruppenangeboten wie Elternabenden gerade in diesen Familien niedrig ist, kann die Schwierigkeit, auch die Eltern dieser Kinder durch geeignete Elternarbeit mit ins Boot zu bekommen, vermutlich eher durch Angebote individueller Erziehungshilfen angegangen werden. Letztlich stellt sich aber auch die Frage, ob medienpädagogischer Unterricht im Sinne einer primären gegenüber einer sekundären Prävention nicht stärker gruppenspezifisch nach Kindern mit problematischem Mediennutzungsverhalten und Kindern ohne problematisches Mediennutzugsverhalten ausgerichtet sein muss, allein schon um Effekten des Gruppendrucks, durch welchen problematische Mediennutzung als erstrebenswert erscheinen könnte, entgegenzuwirken. Eine derartige Aufteilung würde beim momentanen Kenntnisstand allerdings schon weitgehend durch eine geschlechtsspezifische Medienintervention gewährleistet werden können. 